\title{
Generalized parameter-free duality models in discrete minmax fractional programming based on second-order optimality conditions
}

\author{
G. J. Zalmai ${ }^{1}$ Ram U. Verma ${ }^{2}$
}

Received: 4 August 2015/Accepted: 12 October 2016/Published online: 8 November 2016

(C) The Author(s) 2016. This article is published with open access at Springerlink.com

\begin{abstract}
In this paper, we construct six generalized second-order parameter-free duality models, and prove several weak, strong, and strict converse duality theorems for a discrete minmax fractional programming problem using two partitioning schemes and various types of generalized second-order $(\mathcal{F}, \beta, \phi, \rho, \theta, m)$-univexity (more compactly, 'second-order univexity' is referred to as 'sounivexity') assumptions. The obtained results are new and generalize most of results on discrete minmax fractional programming involving the second-order invexity as well as on secondorder univexity in the literature.
\end{abstract}

Keywords Discrete minmax fractional programming . Generalized second-order $(\mathcal{F}, \beta, \phi, \rho, \theta, m)$-univex . Generalized parameter-free duality models · Duality theorems

Mathematics Subject Classification 90C26 - 90C30 . $90 \mathrm{C} 32 \cdot 90 \mathrm{C} 46 \cdot 90 \mathrm{C} 47$

Ram U. Verma

verma99@msn.com

G. J. Zalmai

gzalmai@nmu.edu

1 Department of Mathematics and Computer Science, Northern Michigan University, Marquette, MI 49855, USA

2 Department of Mathematics, University of North Texas, Denton, TX 76201, USA

\section{Introduction and preliminaries}

Based on a close observation on second-order necessary and sufficient optimality conditions for minmax fractional programming problems, which have not received much attention in the literature of mathematical programming, that is in sharp contrast to the case of minmax programming problems, numerous second-order necessary and sufficient optimality conditions for various classes of nonlinear programming problems with single and multiple objective functions have been investigated in the literature, including [1, 8-11, 13, 15, 19-21]. However, none of the sufficient optimality conditions discussed in these publications involve developing any kind of second-order duality theory for any type of optimization problems. The notion of duality for generalized linear fractional programming was initially considered by von Neumann [14] to the context of an economic equilibrium problem. However, a significant number of optimality criteria, duality results, and computational algorithms for several classes of generalized linear and nonlinear fractional programming problems have appeared in the related literature, for example in the publications $[2-7,12,14,16-18$, 22-25]. Verma and Zalmai [10] dealt with some details on discrete minmax fractional programming, a fairly extensive list of currently available publications dealing with various second-order necessary and sufficient optimality conditions for several types of optimization problems, some modifications of the concepts of second-order invexity, pseudoinvexity, and quasiinvexity originally defined by Hanson [3], a set of second-order necessary optimality conditions, and making use of the new classes of generalized secondorder invex functions, a fairly large number of sets of second-order sufficient optimality criteria. The sufficient optimality conditions established in [10] are further 
generalized in [12] using various generalized second-order $(\phi, \eta, \rho, \theta, m)$-invexity assumptions. For more details on generalized linear and nonlinear fractional programming problems, we refer the reader [1-25].

In this paper, it is our intention to lay the theoretical foundation which will enable us to fully investigate the second-order optimality and duality aspects of our following principal problem $(P)$ as well as its semiinfinite counterpart in a series of papers. We begin our investigation here by establishing a set of second-order parametric necessary optimality conditions and several sets of sufficient optimality conditions for principal problem $(P)$. Furthermore, we utilize two partitioning schemes due to Mond and Weir [7] and Yang [18], in conjunction with the generalized versions of the new classes of second-order invex functions introduced in (Verma and Zalmai [10]) to formulate six generalized parameter-free duality models for principal problem $(P)$ and prove appropriate duality theorems. The duality models and the related duality theory established in this paper generalize most of results available in the literature, including those results published in [2-7, 12, 16, 18, 22-25].

To the best of our knowledge, all of these duality results established in this paper are new in the area of discrete minmax fractional programming. In fact, it seems that results of this type, which are based on second-order necessary and sufficient optimality conditions, have not yet appeared in any shape or form for any type of mathematical programming problems in the literature.

Now, we formulate six generalized second-order parameter-free duality models and prove a variety of weak, strong, and strict converse duality theorems for the following discrete minmax fractional programming problem:

$$
\text { (P) Minimize } \max _{1 \leq i \leq p} \frac{f_{i}(x)}{g_{i}(x)}
$$

subject to $G_{j}(x) \leq 0, j \in \underline{q}, H_{k}(x)=0, k \in \underline{r}, x \in X$,

where $x$ is an open convex subset of $\mathbb{R}^{n}$ (n-dimensional Euclidean space), $f_{i}, g_{i}, i \in \underline{p} \equiv\{1,2, \ldots, p\}, G_{j}, j \in \underline{q}$, and $H_{k}, k \in \underline{r}$, are real-valued functions defined on $X$, and for each $i \in p, g_{i}(x)>0$ for all $x$ satisfying the constraints of $(P)$.

Evidently, all the duality results established in this paper can be modified and restated for each one of the following three classes of nonlinear programming problems, which are special cases of $(P)$ :

$$
\begin{aligned}
& (P 1) \quad \text { Minimize } \frac{f_{1}(x)}{g_{1}(x)} ; \\
& (P 2) \quad \text { Minimize } \max _{x \in \mathbb{F}} f_{i}(x) ; \\
& (P 3) \quad \text { Minimize } f_{x \in \mathbb{F}}(x),
\end{aligned}
$$

where $\mathbb{F}$ (assumed to be nonempty) is the feasible set of $(P)$, that is,

$$
\mathbb{F}=\left\{x \in X: G_{j}(x) \leq 0, j \in \underline{q}, H_{k}(x)=0, k \in \underline{r}\right\} .
$$

Since, in most cases, these results can easily be altered and rephrased for each one of the above three problems, we shall not state them explicitly.

The rest of this paper is organized as follows: In Sect. 1, we present the historical development and introduce/recall a few basic definitions and auxiliary results that will be used in the sequel. In Sect. 2, we utilize a partitioning scheme due to Mond and Weir [7], and formulate two general second-order parameter-free duality models for $(P)$ and prove weak, strong, and strict converse duality theorems using various generalized $(\mathcal{F}, \beta, \phi, \rho, \theta, m)$ sounivexity assumptions. We continue our discussion of duality in Sects. 3 and 4 where we construct four additional general second-order parameter-free duality models with different constraint structures and prove several secondorder duality results under a variety of generalized $(\mathcal{F}, \beta, \phi, \rho, \theta, m)$-sounivexity conditions. Finally, in Sect. 5 , we summarize our main results and also point out some research opportunities arising from certain modifications of the principal minmax model investigated in this study.

We next introduce the new classes of "second-order univex' functions (referred to as "sounivex" functions). The notion of 'sounivexity' generalizes the notion of 'second-order invexity,' which is referred to as "sonvexity" in the literature. Let $f: X \rightarrow \mathbb{R}$ be a twice differentiable function.

Definition 1.1 The function $f$ is said to be (strictly) $(\mathcal{F}, \beta, \phi, \rho, \theta, m)$-sounivex at $x^{*}$ if there exist functions $\beta: X \times X \rightarrow \mathbb{R}_{+} \equiv(0, \infty), \phi: \mathbb{R} \rightarrow \mathbb{R}, \rho: X \times X \rightarrow \mathbb{R}, \theta:$ $X \times X \rightarrow \mathbb{R}^{n}$, a sublinear function $\mathcal{F}\left(x, x^{*} ; \cdot\right): \mathbb{R}^{n} \rightarrow \mathbb{R}$, and a positive integer $m$, such that for each $x \in X\left(x \neq x^{*}\right)$ and $z \in \mathbb{R}^{n}$,

$$
\begin{aligned}
\phi\left(f(x)-f\left(x^{*}\right)\right)(>) \geq & \mathcal{F}\left(x, x^{*} ; \beta\left(x, x^{*}\right) \nabla f\left(x^{*}\right)\right) \\
& +\frac{1}{2}\left\langle z, \nabla^{2} f\left(x^{*}\right) z\right\rangle+\rho\left(x, x^{*}\right)\left\|\theta\left(x, x^{*}\right)\right\|^{m},
\end{aligned}
$$

where $\|\cdot\|$ is a norm on $\mathbb{R}^{n}$ and $\langle a, b\rangle$ is the inner product of the vectors $a$ and $b$.

The function $f$ is said to be (strictly) $(\mathcal{F}, \beta, \phi, \rho, \theta, m)$ sounivex on $X$ if it is (strictly) $(\mathcal{F}, \beta, \phi, \rho, \theta, m)$-sounivex at each $x^{*} \in X$.

Definition 1.2 The function $f$ is said to be (strictly) $(\mathcal{F}, \beta, \phi, \rho, \theta, m)$-pseudosounivex at $x^{*}$ if there exist functions $\quad \beta: X \times X \rightarrow \mathbb{R}_{+}, \phi: \mathbb{R} \rightarrow \mathbb{R}, \rho: X \times X \rightarrow \mathbb{R}, \theta:$ $X \times X \rightarrow \mathbb{R}^{n}$, a sublinear function $\mathcal{F}\left(x, x^{*} ; \cdot\right): \mathbb{R}^{n} \rightarrow \mathbb{R}$, and a positive integer $m$, such that for each $x \in X\left(x \neq x^{*}\right)$ and $z \in \mathbb{R}^{n}$, 


$$
\begin{aligned}
& \mathcal{F}\left(x, x^{*} ; \beta\left(x, x^{*}\right) \nabla f\left(x^{*}\right)\right)+\frac{1}{2}\left\langle z, \nabla^{2} f\left(x^{*}\right) z\right\rangle \\
& \quad \geq-\rho\left(x, x^{*}\right)\left\|\theta\left(x, x^{*}\right)\right\|^{m} \\
& \quad \Rightarrow \phi\left(f(x)-f\left(x^{*}\right)\right)(>) \geq 0 .
\end{aligned}
$$

The function $f$ is said to be (strictly) $(\mathcal{F}, \beta, \phi, \rho, \theta, m)$ pseudosounivex on $X$ if it is (strictly) $(\mathcal{F}, \beta, \phi, \rho, \theta, m)$ pseudosounivex at each $x^{*} \in X$.

Definition 1.3 The function $f$ is said to be (prestrictly) $(\mathcal{F}, \beta, \phi, \rho, \theta, m)$-quasisounivex at $x^{*}$ if there exist functions $\quad \beta: X \times X \rightarrow \mathbb{R}_{+}, \phi: \mathbb{R} \rightarrow \mathbb{R}, \rho: X \times X \rightarrow \mathbb{R}, \theta:$ $X \times X \rightarrow \mathbb{R}^{n}$, a sublinear function $\mathcal{F}\left(x, x^{*} ; \cdot\right): \mathbb{R}^{n} \rightarrow \mathbb{R}$, and a positive integer $m$, such that for each $x \in X$ and $z \in \mathbb{R}^{n}$

$$
\begin{aligned}
& \phi\left(f(x)-f\left(x^{*}\right)\right)(<) \leq 0 \\
& \quad \Rightarrow \mathcal{F}\left(x, x^{*} ; \beta\left(x, x^{*}\right) \nabla f\left(x^{*}\right)\right)+\frac{1}{2}\left\langle z, \nabla^{2} f\left(x^{*}\right) z\right\rangle \\
& \quad \leq-\rho\left(x, x^{*}\right)\left\|\theta\left(x, x^{*}\right)\right\|^{m} .
\end{aligned}
$$

The function $f$ is said to be (prestrictly) $(\mathcal{F}, \beta, \phi, \rho, \theta, m)$ quasisounivex on $X$ if it is (prestrictly) $(\mathcal{F}, \beta, \phi, \rho, \theta, m)$ quasisounivex at each $x^{*} \in X$.

From the above definitions, it is clear that if $f$ is $(\mathcal{F}, \beta, \phi, \rho, \theta, m)$-sounivex at $x^{*}$, then it is both $(\mathcal{F}, \beta, \phi, \rho, \theta, m)$-pseudosounivex and $(\mathcal{F}, \beta, \phi, \rho, \theta, m)$ quasisounivex at $x^{*}$, if $f$ is $(\mathcal{F}, \beta, \phi, \rho, \theta, m)$-quasisounivex at $x^{*}$, then it is prestrictly $(\mathcal{F}, \beta, \phi, \rho, \theta, m)$-quasisounivex at $x^{*}$, and if $f$ is strictly $(\mathcal{F}, \beta, \phi, \rho, \theta, m)$-pseudosounivex at $x^{*}$, then it is $(\mathcal{F}, \beta, \phi, \rho, \theta, m)$-quasisounivex at $x^{*}$.

In the proofs of the duality theorems, sometimes, it may be more convenient to use certain alternative but equivalent forms of the above definitions. These are obtained by considering the contrapositive statements. For example, $(\mathcal{F}, \beta, \phi, \rho, \theta, m)$-quasisounivexity can be defined in the following equivalent way:

The function $f$ is said to be $(\mathcal{F}, \beta, \phi, \rho, \theta, m)$-quasisounivex at $x^{*}$ if there exist functions $\beta: X \times X \rightarrow$ $\mathbb{R}_{+}, \phi: \mathbb{R} \rightarrow \mathbb{R}, \rho: X \times X \rightarrow \mathbb{R}, \theta: X \times X \rightarrow \mathbb{R}^{n}$, a sublinear function $\mathcal{F}\left(x, x^{*} ; \cdot\right): \mathbb{R}^{n} \rightarrow \mathbb{R}$, and a positive integer $m$, such that for each $x \in X$ and $z \in \mathbb{R}^{n}$,

$$
\begin{aligned}
& \mathcal{F}\left(x, x^{*} ; \beta\left(x, x^{*}\right) \nabla f\left(x^{*}\right)\right)+\frac{1}{2}\left\langle z, \nabla^{2} f\left(x^{*}\right) z\right\rangle \\
& >-\rho\left(x, x^{*}\right)\left\|\theta\left(x, x^{*}\right)\right\|^{m} \\
& \quad \Rightarrow \phi\left(f(x)-f\left(x^{*}\right)\right)>0 .
\end{aligned}
$$

Needless to say that the new classes of generalized convex functions specified in Definitions 1.1-1.3 contain a variety of special cases that can easily be identified by appropriate choices of $\mathcal{F}, \beta, \phi, \rho, \theta$, and $m$. For example, if let $\mathcal{F}\left(x, x^{*} ; \nabla f\left(x^{*}\right)\right)=\left\langle\nabla f\left(x^{*}\right), \eta\left(x, x^{*}\right)\right\rangle \quad$ and $\quad \beta\left(x, x^{*}\right) \equiv 1$, then we obtain the definitions of (strictly) $(\phi, \eta, \rho, \theta, m)$ - sonvex, (strictly) $(\phi, \eta, \rho, \theta, m)$-pseudosonvex, and (prestrictly) $(\phi, \eta, \rho, \theta, m)$-quasisonvex functions introduced recently in [10], where the "second-order invexity" is compactly abbreviated as "sonvexity." The notion of the sonvexity/generalized sonvexity has been applied in developing a new optimality-duality theory in nonlinear programming based on second-order necessary and sufficient optimality conditions $[1,8-10,12,22]$.

Definition 1.4 The function $f$ is said to be (strictly) $(\phi, \eta, \rho, \theta, m)$-sonvex at $x^{*}$ if there exist functions $\phi: \mathbb{R} \rightarrow \mathbb{R}, \eta: X \times X \rightarrow \mathbb{R}^{n}, \rho: X \times X \rightarrow \mathbb{R}$, and $\theta: X \times$ $X \rightarrow \mathbb{R}^{n}$, and a positive integer $m$, such that for each $x \in$ $X\left(x \neq x^{*}\right)$ and $z \in \mathbb{R}^{n}$,

$$
\begin{aligned}
\phi\left(f(x)-f\left(x^{*}\right)\right)(>) \geq & \left\langle\nabla f\left(x^{*}\right), \eta\left(x, x^{*}\right)\right\rangle+\frac{1}{2}\left\langle z, \nabla^{2} f\left(x^{*}\right) z\right\rangle \\
& +\rho\left(x, x^{*}\right)\left\|\theta\left(x, x^{*}\right)\right\|^{m} .
\end{aligned}
$$

The function $f$ is said to be (strictly) $(\phi, \eta, \rho, \theta, m)$-sonvex on $X$ if it is (strictly) $(\phi, \eta, \rho, \theta, m)$-sonvex at each $x^{*} \in X$.

Definition 1.5 The function $f$ is said to be (strictly) $(\phi, \eta, \rho, \theta, m)$-pseudosonvex at $x^{*}$ if there exist functions $\phi: \mathbb{R} \rightarrow \mathbb{R}, \eta: X \times X \rightarrow \mathbb{R}^{n}, \rho: X \times X \rightarrow \mathbb{R}$, and $\theta: X \times$ $X \rightarrow \mathbb{R}^{n}$, and a positive integer $m$, such that for each $x \in$ $X\left(x \neq x^{*}\right)$ and $z \in \mathbb{R}^{n}$,

$$
\begin{aligned}
& \left\langle\nabla f\left(x^{*}\right), \eta\left(x, x^{*}\right)\right\rangle+\frac{1}{2}\left\langle z, \nabla^{2} f\left(x^{*}\right) z\right\rangle \geq-\rho\left(x, x^{*}\right)\left\|\theta\left(x, x^{*}\right)\right\|^{m} \\
& \quad \Rightarrow \phi\left(f(x)-f\left(x^{*}\right)\right)(>) \geq 0
\end{aligned}
$$

equivalently,

$$
\begin{aligned}
\phi\left(f(x)-f\left(x^{*}\right)\right)(\leq)<0 & \Rightarrow\left\langle\nabla f\left(x^{*}\right), \eta\left(x, x^{*}\right)\right\rangle+\frac{1}{2}\left\langle z, \nabla^{2} f\left(x^{*}\right) z\right\rangle \\
& <-\rho\left(x, x^{*}\right)\left\|\theta\left(x, x^{*}\right)\right\|^{m} .
\end{aligned}
$$

The function $f$ is said to be (strictly) $(\phi, \eta, \rho, \theta, m)$-pseudosonvex on $X$ if it is (strictly) $(\phi, \eta, \rho, \theta, m)$-pseudosonvex at each $x^{*} \in X$.

Definition 1.6 The function $f$ is said to be (prestrictly) $(\phi, \eta, \rho, \theta, m)$-quasisonvex at $x^{*}$ if there exist functions $\phi: \mathbb{R} \rightarrow \mathbb{R}, \eta: X \times X \rightarrow \mathbb{R}^{n}, \rho: X \times X \rightarrow \mathbb{R}$, and $\theta: X \times$ $X \rightarrow \mathbb{R}^{n}$, and a positive integer $m$, such that for each $x \in X$ and $z \in \mathbb{R}^{n}$,

$$
\begin{gathered}
\phi\left(f(x)-f\left(x^{*}\right)\right)(<) \leq 0 \Rightarrow\left\langle\nabla f\left(x^{*}\right), \eta\left(x, x^{*}\right)\right\rangle \\
+\frac{1}{2}\left\langle z, \nabla^{2} f\left(x^{*}\right) z\right\rangle \leq-\rho\left(x, x^{*}\right)\left\|\theta\left(x, x^{*}\right)\right\|^{m},
\end{gathered}
$$

equivalently

$$
\begin{aligned}
& \left\langle\nabla f\left(x^{*}\right), \eta\left(x, x^{*}\right)\right\rangle+\frac{1}{2}\left\langle z, \nabla^{2} f\left(x^{*}\right) z\right\rangle>-\rho\left(x, x^{*}\right)\left\|\theta\left(x, x^{*}\right)\right\|^{m} \\
& \quad \Rightarrow \phi\left(f(x)-f\left(x^{*}\right)\right)(\geq)>0 .
\end{aligned}
$$


The function $f$ is said to be (prestrictly) $(\phi, \eta, \rho, \theta, m)$ quasisonvex on $X$ if it is (prestrictly) $(\phi, \eta, \rho, \theta, m)$-quasisonvex at each $x^{*} \in X$.

\section{Duality model I and duality theorems}

We begin this section by recalling a set of second-order parameter-free necessary optimality conditions for $(P)$. This result, which is obtained from Theorem 3.1 of [10] by eliminating the parameter $\lambda^{*}$ and redefining the Lagrange multipliers, will be needed for proving strong and strict converse duality theorems.

Theorem 2.1 [10] Let $x^{*}$ be a normal optimal solution of $(P)$ and assume that the functions $f_{i}, g_{i}, i \in \underline{p}, G_{j}, j \in \underline{q}$, and $H_{k}, k \in \underline{r}$, are twice continuously differentiable at $x^{*}$. Then, for each $z^{*} \in C\left(x^{*}\right)$, there exist $u^{*} \in U \equiv\left\{u \in \mathbb{R}^{p}\right.$ : $\left.u \geq 0, \sum_{i=1}^{p} u_{i}=1\right\}, v^{*} \in \mathbb{R}_{+}^{q} \equiv\left\{v \in \mathbb{R}^{q}: v \geq 0\right\}, \quad$ and $w^{*} \in \mathbb{R}^{r}$, such that

$$
\begin{aligned}
& \sum_{i=1}^{p} u_{i}^{*}\left[D\left(x^{*}, u^{*}\right) \nabla f_{i}\left(x^{*}\right)-N\left(x^{*}, u^{*}\right) \nabla g_{i}\left(x^{*}\right)\right] \\
& \quad+\sum_{j=1}^{q} v_{j}^{*} \nabla G_{j}\left(x^{*}\right)+\sum_{k=1}^{r} w_{k}^{*} \nabla H_{k}\left(x^{*}\right)=0, \\
& \left\langle z^{*},\left\{\sum_{i=1}^{p} u_{i}^{*}\left[D\left(x^{*}, u^{*}\right) \nabla^{2} f_{i}\left(x^{*}\right)-N\left(x^{*}, u^{*}\right) \nabla^{2} g_{i}\left(x^{*}\right)\right]\right.\right. \\
& \left.\left.\quad+\sum_{j=1}^{q} v_{j}^{*} \nabla^{2} G_{j}\left(x^{*}\right)+\sum_{k=1}^{r} w_{k}^{*} \nabla^{2} H_{k}\left(x^{*}\right)\right\} z^{*}\right\rangle \geq 0, \\
& u_{i}^{*}\left[D\left(x^{*}, u^{*}\right) f_{i}\left(x^{*}\right)-N\left(x^{*}, u^{*}\right) g_{i}\left(x^{*}\right)\right]=0, i \in \underline{p}, \\
& \max _{1 \leq i \leq p} \frac{f_{i}\left(x^{*}\right)}{g_{i}\left(x^{*}\right)}=\frac{N\left(x^{*}, u^{*}\right)}{D\left(x^{*}, u^{*}\right)}, \\
& v_{j}^{*} G_{j}\left(x^{*}\right)=0, j \in \underline{q},
\end{aligned}
$$

where $C\left(x^{*}\right)$ is the set of all critical directions of $(P)$ at $x^{*}$, that is

$$
\begin{aligned}
C\left(x^{*}\right) & =\left\{z^{*} \in \mathbb{R}^{n}:\left\langle D\left(x^{*}, u^{*}\right) \nabla f_{i}\left(x^{*}\right)-N\left(x^{*}, u^{*}\right) g_{i}\left(x^{*}\right), z^{*}\right\rangle=0,\right. \\
i \in & \left.A\left(x^{*}\right),\left\langle\nabla G_{j}\left(x^{*}\right), z^{*}\right\rangle \leq 0, j \in B\left(x^{*}\right),\left\langle\nabla H_{k}\left(x^{*}\right), z^{*}\right\rangle=0, k \in \underline{r}\right\}, \\
A\left(x^{*}\right) & =\left\{j \in \underline{p}: f_{j}\left(x^{*}\right) / g_{j}\left(x^{*}\right)=\max _{1 \leq i \leq p} f_{i}\left(x^{*}\right) / g_{i}\left(x^{*}\right)\right\}, B\left(x^{*}\right) \\
& =\left\{j \in \underline{q}: G_{j}\left(x^{*}\right)=0\right\}, N\left(x^{*}, u^{*}\right)=\sum_{i=1}^{p} u_{i}^{*} f_{i}\left(x^{*}\right), \text { and } D\left(x^{*}, u^{*}\right) \\
& =\sum_{i=1}^{p} u_{i}^{*} g_{i}\left(x^{*}\right) .
\end{aligned}
$$

In the above theorem, a normal optimal solution refers to an optimal solution at which an appropriate second-order constraint qualification is satisfied.
In the remainder of this paper, we shall assume that the functions $f_{i}, g_{i}, i \in \underline{p}, G_{j}, j \in \underline{q}$, and $H_{k}, k \in \underline{r}$, are twice continuously differentiable on the open set $X$. Moreover, we shall assume, without loss of generality, that for each $i \in \underline{p}, f_{i}(x) \geq 0$ and $g_{i}(x)>0$ for all $x \in X$.

\section{Duality model I}

In this section, we discuss several families of duality results under various generalized $(\mathcal{F}, \beta, \phi, \rho, \theta, m)$ sounivexity hypotheses imposed on certain combinations of the problem functions. This is accomplished by employing a certain partitioning scheme which was originally proposed in [7] for the purpose of constructing generalized dual problems for nonlinear programming problems. For this, we need some additional notation.

Let $\left\{J_{0}, J_{1}, \ldots, J_{M}\right\}$ and $\left\{K_{0}, K_{1}, \ldots, K_{M}\right\}$ be partitions of the index sets $q$ and $\underline{r}$, respectively; thus, $J_{\mu} \subseteq \underline{q}$ for each $\mu \in \underline{M} \cup\{0\}, J_{\mu} \cap J_{v}=\emptyset$ for each $\mu, v \in \underline{M} \cup\{0\}$ with $\mu \neq v$, and $\cup_{\mu=0}^{M} J_{\mu}=\underline{q}$. Obviously, similar properties hold for $\left\{K_{0}, K_{1}, \ldots, K_{M}\right\}$. Moreover, if $m_{1}$ and $m_{2}$ are the numbers of the partitioning sets of $q$ and $\underline{r}$, respectively, then $M=\max \left\{m_{1}, m_{2}\right\} \quad$ and $J_{\mu}=\emptyset \quad$ or $\quad K_{\mu}=\emptyset$ for $\mu>\min \left\{m_{1}, m_{2}\right\}$.

In addition, we use the real-valued functions $\xi \rightarrow \Phi(\xi, u, v, w, \lambda)$, and $\xi \rightarrow \Lambda_{t}(\xi, v, w)$ defined, for fixed $\lambda, u, v$, and $w$, on $X$ as follows:

$$
\begin{aligned}
\Phi(\xi, y, u, v, w)= & \sum_{i=1}^{p} u_{i}\left\{D(y, u)\left[f_{i}(\xi)+\sum_{j \in J_{0}} v_{j} G_{j}(\xi)+\sum_{k \in K_{0}} w_{k} H_{k}(\xi)\right]\right. \\
& \left.-\left[N(y, u)+\Lambda_{0}(y, v, w)\right] g_{i}(\xi)\right\}, \\
\Lambda_{t}(\xi, v, w)= & \sum_{j \in J_{t}} v_{j} G_{j}(\xi)+\sum_{k \in K_{t}} w_{k} H_{k}(\xi), \quad t \in \underline{M} \cup\{0\} .
\end{aligned}
$$

Making use of the sets and functions defined above, we can now formulate our first pair of second-order parameter-free duality models for $(P)$.

Consider the following two problems:

$(D I)$

$\frac{\sum_{i=1}^{p} u_{i} f_{i}(y)+\sum_{j \in J_{0}} v_{j} G_{j}(y)+\sum_{k \in K_{0}} w_{k} H_{k}(y)}{\sum_{i=1}^{p} u_{i} g_{i}(y)}$

Maximize

subject to

$$
\begin{aligned}
& \sum_{i=1}^{p} u_{i}\left\{D(y, u)\left[\nabla f_{i}(y)+\sum_{j \in J_{0}} v_{j} \nabla G_{j}(y)+\sum_{k \in K_{0}} w_{k} \nabla H_{k}(y)\right]\right. \\
& \left.-\left[N(y, u)+\Lambda_{0}(y, v, w)\right] \nabla g_{i}(y)\right\}+\sum_{j \in \underline{q} \backslash J_{0}} v_{j} \nabla G_{j}(y) \\
& \quad+\sum_{k \in \underline{r} \backslash K_{0}} w_{k} \nabla H_{k}(y)=0
\end{aligned}
$$




$$
\begin{aligned}
& \left\langle z, \sum_{i=1}^{p} u_{i}\left\{D(y, u)\left[\nabla^{2} f_{i}(y)+\sum_{j \in J_{0}} v_{j} \nabla^{2} G_{j}(y)+\sum_{k \in K_{0}} w_{k} \nabla^{2} H_{k}(y)\right]\right.\right. \\
& \left.\quad-\left[N(y, u)+\Lambda_{0}(y, v, w)\right] \nabla^{2} g_{i}(y)\right\}+\sum_{j \in \underline{q} \backslash J_{0}} v_{j} \nabla^{2} G_{j}(y) \\
& \left.\left.\quad+\sum_{k \in \underline{r} \backslash K_{0}} w_{k} \nabla^{2} H_{k}(y)\right\} z\right\rangle \geq 0 \\
& \sum_{j \in J_{t}} v_{j} G_{j}(y)+\sum_{k \in K_{t}} w_{k} H_{k}(y) \geq 0, t \in \underline{M} \\
& y \in X, z \in C(y), u \in U, v \in \mathbb{R}_{+}^{q}, w \in \mathbb{R}^{r}
\end{aligned}
$$

$(\tilde{D} I)$ Maximize

$\frac{\sum_{i=1}^{p} u_{i} f_{i}(y)+\sum_{j \in J_{0}} v_{j} G_{j}(y)+\sum_{k \in K_{0}} w_{k} H_{k}(y)}{\sum_{i=1}^{p} u_{i} g_{i}(y)}$

subject to (2.2)-(2.4) and

$$
\begin{gathered}
\mathcal{F}\left(x, y ; \sum_{i=1}^{p} u_{i}\left\{D(y, u)\left[\nabla f_{i}(y)+\sum_{j \in J_{0}} v_{j} \nabla G_{j}(y)+\sum_{k \in K_{0}} w_{k} \nabla H_{k}(y)\right]\right.\right. \\
\left.-\left[N(y, u)+\Lambda_{0}(y, v, w)\right] \nabla g_{i}(y)\right\}+\sum_{j \in \underline{q} \backslash J_{0}} v_{j} \nabla G_{j}(y)
\end{gathered}
$$$$
\left.+\sum_{k \in \underline{r} \backslash K_{0}} w_{k} \nabla H_{k}(y)\right) \geq 0 \text { for all } x \in \mathbb{F},
$$

where $\mathcal{F}(x, y ; \cdot)$ is a sublinear function from $\mathbb{R}^{n}$ to $\mathbb{R}$.

Comparing (DI) and $(\tilde{D} I)$, we see that $(\tilde{D} I)$ is relatively more general than (DI) in the sense that any feasible solution of (DI) is also feasible for $(\tilde{D} I)$, but the converse is not necessarily true. Furthermore, we observe that (2.1) is a system of $n$ equations, whereas (2.5) is a single inequality. Clearly, from a computational point of view, $(D I)$ is preferable to $(\tilde{D} I)$ because of the dependence of (2.5) on the feasible set of $(P)$.

Despite these apparent differences, it turns out that the statements and proofs of all the duality theorems for $(P)-(D I)$ and $(P)-(\tilde{D} I)$ are almost identical and, therefore, we shall consider only the pair $(P)-(D I)$.

In the proofs of our duality theorems, we shall make frequent use of the following auxiliary result which provides an alternative expression for the objective function of $(P)$.

Lemma 2.1 [8] For each $x \in X$,

$$
\varphi(x)=\max _{1 \leq i \leq p} \frac{f_{i}(x)}{g_{i}(x)}=\max _{u \in U} \frac{\sum_{i=1}^{p} u_{i} f_{i}(x)}{\sum_{i=1}^{p} u_{i} g_{i}(x)} .
$$

The next two theorems show that (DI) is a dual problem for $(P)$.

Theorem 2.2 (Weak duality) Let $x$ and $\mathcal{S} \equiv(y, z, u, v, w)$ be arbitrary feasible solutions of $(P)$ and $(D I)$, respectively, and assume that any one of the following four sets of hypotheses is satisfied:

1.

(a) $\xi \rightarrow \Phi(\xi, y, u, v, w)$ is $(\mathcal{F}, \beta, \bar{\phi}, \bar{\rho}, \theta, m)$-pseudosounivex at $y$ and $\bar{\phi}(a) \geq 0 \Rightarrow a \geq 0$;

(b) For each $t \in \underline{M}, \xi \rightarrow \Lambda_{t}(z, v, w)$ is $\left(\mathcal{F}, \beta, \tilde{\phi}_{t}\right.$, $\left.\tilde{\rho}_{t}, \theta, m\right)$-quasisounivex at $y, \tilde{\phi}_{t}$ is increasing, and $\tilde{\phi}_{t}(0)=0$

(c) $\bar{\rho}(x, y)+\sum_{t=1}^{M} \tilde{\rho}_{t}(x, y) \geq 0$;

2.

(a) $\xi \rightarrow \Phi(\xi, y, u, v, w)$ is prestrictly $(\mathcal{F}, \beta, \bar{\phi}, \bar{\rho}$, $\theta, m)$-quasisounivex at $y$ and $\bar{\phi}(a) \geq 0 \Rightarrow a \geq 0$;

(b) for each $t \in \underline{M}, \xi \rightarrow \Lambda_{t}(\xi, v, w)$ is $\left(\mathcal{F}, \beta, \tilde{\rho}_{t}\right.$, $\left.\rho_{t}, \theta, m\right)$-quasisounivex at $y, \tilde{\phi}_{t}$ is increasing, and $\tilde{\phi}_{t}(0)=0$

(c) $\bar{\rho}(x, y)+\sum_{t=1}^{M} \tilde{\rho}_{t}(x, y)>0$;

3.

(a) $\xi \rightarrow \Phi(\xi, y, u, v, w)$ is prestrictly $(\mathcal{F}, \beta, \bar{\phi}, \bar{\rho}$, $\theta, m)$-quasisounivex at $y, \bar{\phi}$ is strictly increasing, and $\bar{\phi}(0)=0$

(b) For each $t \in \underline{M}, \xi \rightarrow \Lambda_{t}(\xi, v, w)$ is strictly $\left(\mathcal{F}, \beta, \tilde{\phi}_{t}, \tilde{\rho}_{t}, \theta, m\right)$-pseudosounivex at $y, \tilde{\phi}_{t}$ is increasing, and $\tilde{\phi}_{t}(0)=0$;

(c) $\bar{\rho}(x, y)+\sum_{t=1}^{M} \tilde{\rho}_{t}(x, y) \geq 0$;

4.

(a) $\xi \rightarrow \Phi(\xi, y, u, v, w)$ is prestrictly $(\mathcal{F}, \beta, \bar{\phi}, \bar{\rho}$, $\theta, m)$-quasisounivex at $y, \bar{\phi}$ is strictly increasing, and $\bar{\phi}(0)=0$;

(b) For each $t \in \underline{M_{1}}, \xi \rightarrow \Lambda_{t}(\xi, v, w)$ is $\left(\mathcal{F}, \beta, \tilde{\phi}_{t}, \tilde{\rho}_{t}\right.$, $\theta, m)$-quasisounivex at $y$, for each $t \in \underline{M_{2}} \neq$ $\emptyset, \xi \rightarrow \Lambda_{t}(\xi, v, w)$ is strictly $\left(\mathcal{F}, \beta, \tilde{\phi}_{t}, \tilde{\rho}_{t}, \overline{\theta, m}\right)$ pseudosounivex at $y$, and for each $t \in \underline{M}, \tilde{\phi}_{t}$ is increasing and $\tilde{\phi}_{t}(0)=0$, where $\left\{M_{1}, M_{2}\right\}$ is a partition of $\underline{M}$;

(c) $\bar{\rho}(x, y)+\sum_{t=1}^{M} \tilde{\rho}_{t}(x, y) \geq 0$.

Then, $\varphi(x) \geq \psi_{I}(y, u, v, w)$, where $\psi_{I}$ is the objective function of $(D I)$.

Proof (a): Since $\mathcal{F}(x, y ; \cdot)$ is sublinear and $\beta(x, y)>0$, it is clear that (2.1) and (2.2) can be expressed as follows: 


$$
\begin{aligned}
& \mathcal{F}\left(x, y ; \beta(x, y) \sum_{i=1}^{p} u_{i}\left\{D ( y , u ) \left[\nabla f_{i}(y)+\sum_{j \in J_{0}} v_{j} \nabla G_{j}(y)\right.\right.\right. \\
&\left.\left.\left.+\sum_{k \in K_{0}} w_{k} \nabla H_{k}(y)\right]-\left[N(y, u)+\Lambda_{0}(y, v, w)\right] \nabla g_{i}(y)\right\}\right) \\
&+\mathcal{F}\left(x, y ; \beta(x, y) \sum_{t=1}^{M}\left[\sum_{j \in J_{t}} v_{j} \nabla G_{j}(y)\right.\right. \\
&\left.\left.+\sum_{k \in K_{t}} w_{k} \nabla H_{k}(y)\right]\right) \geq 0 . \\
&\left\langle z, \sum_{i=1}^{p} u_{i}\left\{D(y, u)\left[\nabla^{2} f_{i}(y)+\sum_{j \in J_{0}} v_{j} \nabla^{2} G_{j}(y)+\sum_{k \in K_{0}} w_{k} \nabla^{2} H_{k}(y)\right]\right.\right. \\
&\left.\left.\quad-\left[N(y, u)+\Lambda_{0}(y, v, w)\right] \nabla^{2} g_{i}(y)\right\} z\right\rangle \\
& \quad+\left\langle z, \sum_{t=1}^{M}\left[\sum_{j \in J_{t}} v_{j} \nabla^{2} G_{j}(y)+\sum_{k \in K_{t}} w_{k} \nabla^{2} H_{k}(y)\right] z\right\rangle \geq 0 .
\end{aligned}
$$

Since for each $t \in \underline{M}$,

$$
\begin{aligned}
\Lambda_{t}(x, v, w)= & \sum_{j \in J_{t}} v_{j} G_{j}(x)+\sum_{k \in K_{t}} w_{k} H_{k}(x) \\
\leq & 0 \text { (by the primal feasibility of } x) \\
\leq & \sum_{j \in J_{t}} v_{j} G_{j}(y)+\sum_{k \in K_{t}} w_{k} H_{k}(y) \\
& (\text { by }(2.3) \text { and the dual feasibility of } \mathcal{S}) \\
= & \Lambda_{t}(y, v, w),
\end{aligned}
$$

and hence, $\tilde{\phi}_{t}\left(\Lambda_{t}(x, v, w)-\Lambda_{t}(y, v, w)\right) \leq 0$, it follows from (ii) that

$$
\begin{aligned}
\mathcal{F}\left(x, y ; \beta(x, y)\left[\sum_{j \in J_{t}} v_{j} \nabla G_{j}(y)+\sum_{k \in K_{t}} w_{k} \nabla H_{k}(y)\right]\right) \\
+\frac{1}{2}\left\langle z,\left[\sum_{j \in J_{t}} v_{j} \nabla^{2} G_{j}(y)+\sum_{k \in K_{t}} w_{k} \nabla^{2} H_{k}(y)\right] z\right\rangle \\
\leq-\tilde{\rho}_{t}(x, y)\|\theta(x, y)\|^{m} .
\end{aligned}
$$

Summing over $t \in \underline{M}$ and using the sublinearity of $\mathcal{F}(x, y ; \cdot)$, we obtain

$$
\begin{aligned}
\mathcal{F}\left(x, y ; \beta(x, y) \sum_{t=1}^{M}\left[\sum_{j \in J_{t}} v_{j} \nabla G_{j}(y)+\sum_{k \in K_{t}} w_{k} \nabla H_{k}(y)\right]\right) \\
+\frac{1}{2}\left\langle z, \sum_{t=1}^{M}\left[\sum_{j \in J_{t}} v_{j} \nabla^{2} G_{j}(y)+\sum_{k \in K_{t}} w_{k} \nabla^{2} H_{k}(y)\right] z\right\rangle \\
\leq-\sum_{t=1}^{M} \tilde{\rho}_{t}(x, y)\|\theta(x, y)\|^{m} .
\end{aligned}
$$

Combining (2.6)-(2.8) and using (iii), we get

$$
\begin{aligned}
& \mathcal{F}\left(x, y ; \beta(x, y) \sum_{i=1}^{p} u_{i}\left\{D(y, u)\left[\nabla f_{i}(y)+\sum_{j \in J_{0}} v_{j} \nabla G_{j}(y)+\sum_{k \in K_{0}} w_{k} \nabla H_{k}(y)\right]\right.\right. \\
& \left.\left.\quad-\left[N(y, u)+\Lambda_{0}(y, v, w)\right] \nabla g_{i}(y)\right\}\right)+\frac{1}{2}\left\langle z, \sum_{i=1}^{p} u_{i}\left\{D ( y , u ) \left[\nabla^{2} f_{i}(y)\right.\right.\right. \\
& \left.\left.\left.\quad+\sum_{j \in J_{0}} v_{j} \nabla^{2} G_{j}(y)+\sum_{k \in K_{0}} w_{k} \nabla^{2} H_{k}(y)\right]-\left[N(y, u)+\Lambda_{0}(y, v, w)\right] \nabla^{2} g_{i}(y)\right\} z\right\rangle \\
& \geq \sum_{t=1}^{M} \tilde{\rho}_{t}(x, y)\|\theta(x, y)\|^{m} \geq-\bar{\rho}(x, y)\|\theta(x, y)\|^{m},
\end{aligned}
$$

which by virtue of (i) implies that

$\bar{\phi}(\Phi(x, y, u, v, w)-\Phi(y, y, u, v, w)) \geq 0$.

However, $\bar{\phi}(a) \geq 0 \Rightarrow a \geq 0$, and hence, we get

$\Phi(x, y, u, v, w) \geq \Phi(y, y, u, v, w)=0$,

where the equality follows from the definitions of $D(y, u), N(y, u)$, and $\Lambda_{0}(y, v, w)$. Since $x \in \mathbb{F}$, the above inequality reduces to

$\left.\sum_{i=1}^{p} u_{i}\left\{D(y, u) f_{i}(x)-\left[N(y, u)+\Lambda_{0}(y, v, w)\right] g_{i}(x)\right]\right\} \geq 0$.

Now, using (2.10) and Lemma 2.1, we obtain the weak duality inequality as follows:

$$
\begin{aligned}
\varphi(x) & =\max _{a \in U} \frac{\sum_{i=1}^{p} a_{i} f_{i}(x)}{\sum_{i=1}^{p} a_{i} g_{i}(x)} \geq \frac{\sum_{i=1}^{p} u_{i} f_{i}(x)}{\sum_{i=1}^{p} u_{i} g_{i}(x)} \\
& \geq \frac{N(y, u)+\Lambda_{0}(y, v, w)}{D(y, u)}=\psi_{I}(y, z, u, v, w) .
\end{aligned}
$$

(b) The proof is similar to that of part (a).

(c) Suppose to the contrary that $\varphi(x)<\psi_{I}(y, z, u, v, w)$. This implies that for each $i \in \underline{p}$,

$D(y, u) f_{i}(x)-\left[N(y, u)+\Lambda_{0}(y, v, w)\right] g_{i}(x)<0$.

Using these inequalities, we see that

$$
\begin{aligned}
\Phi(x, y, u, v, w)= & \sum_{i=1}^{p} u_{i}\left\{D ( y , u ) \left[f_{i}(x)+\sum_{j \in J_{0}} v_{j} G_{j}(x)\right.\right. \\
& \left.\left.+\sum_{k \in K_{0}} w_{k} H_{k}(x)\right]-\left[N(y, u)+\Lambda_{0}(y, v, w)\right] g_{i}(x)\right\}, \\
\leq & \sum_{i=1}^{p} u_{i}\left\{D(y, u) f_{i}(x)-\left[N(y, u)+\Lambda_{0}(y, v, w)\right] g_{i}(x)\right\} \\
& \text { (by the primal of feasibility of } x) \\
< & 0(\text { by }(2.11)) \\
= & \Phi(y, y, u, v, w)(\text { by the definitions } \\
& \text { of } \left.D(y, u), N(y, u), \text { and } \Lambda_{0}(y, v, w)\right),
\end{aligned}
$$

and hence, $\bar{\phi}(\Phi(x, y, u, v, w)-\Phi(y, y, u, v, w))<0$ which by virtue of (i) implies that 


$$
\begin{aligned}
\mathcal{F} & \left(x, y ; \beta(x, y) \sum_{i=1}^{p} u_{i}\left\{D(y, u)\left[\nabla f_{i}(y)+\sum_{j \in J_{0}} v_{j} \nabla G_{j}(y)+\sum_{k \in K_{0}} w_{k} \nabla H_{k}(y)\right]\right.\right. \\
- & {\left.\left.\left[N(y, u)+\Lambda_{0}(y, v, w)\right] \nabla g_{i}(y)\right\}\right)+\frac{1}{2}\left\langle z, \sum_{i=1}^{p} u_{i}\left\{D ( y , u ) \left[\nabla^{2} f_{i}(y)\right.\right.\right.} \\
& \left.+\sum_{j \in J_{0}} v_{j} \nabla^{2} G_{j}(y)+\sum_{k \in K_{0}} w_{k} \nabla^{2} H_{k}(y)\right]-[N(y, u) \\
& \left.\left.\left.+\Lambda_{0}(y, v, w)\right] \nabla^{2} g_{i}(y)\right\} z\right\rangle \leq-\bar{\rho}(x, y)\|\theta(x, y)\|^{m} .
\end{aligned}
$$

Proceeding as in the proof of part (a), we obtain $\tilde{\phi}_{t}\left(\Lambda_{t}(x, v, w)-\Lambda_{t}(y, v, w)\right) \leq 0$, which, because of (ii), implies that

$$
\begin{aligned}
& \mathcal{F}\left(x, y ; \beta(x, y)\left[\sum_{j \in J_{t}} v_{j} \nabla G_{j}(y)+\sum_{k \in K_{t}} w_{k} \nabla H_{k}(y)\right]\right)+\frac{1}{2}\left\langle z,\left[\sum_{j \in J_{t}} v_{j} \nabla^{2} G_{j}(y)\right.\right. \\
& \left.\left.\quad+\sum_{k \in K_{t}} w_{k} \nabla^{2} H_{k}(y)\right] z\right\rangle<-\tilde{\rho}_{t}(x, y)\|\theta(x, y)\|^{m} .
\end{aligned}
$$

Summing over $t \in \underline{M}$ and using the sublinearity of $\mathcal{F}(x, y ; \cdot)$, we obtain

$$
\begin{aligned}
\mathcal{F}\left(x, y ; \beta(x, y) \sum_{t=1}^{M}\left[\sum_{j \in J_{t}} v_{j} \nabla G_{j}(y)+\sum_{k \in K_{t}} w_{k} \nabla H_{k}(y)\right]\right) \\
+\frac{1}{2}\left\langle z, \sum_{t=1}^{M}\left[\sum_{j \in J_{t}} v_{j} \nabla^{2} G_{j}(y)+\sum_{k \in K_{t}} w_{k} \nabla^{2} H_{k}(y)\right] z\right\rangle \\
<-\sum_{t=1}^{M} \tilde{\rho}_{t}(x, y)\|\theta(x, y)\|^{m} .
\end{aligned}
$$

Combining this inequality with (2.6) and (2.7) and using (iii), we get

$$
\begin{aligned}
\mathcal{F} & \left(x, y ; \beta(x, y) \sum_{i=1}^{p} u_{i}\left\{D(y, u)\left[\nabla f_{i}(y)+\sum_{j \in J_{0}} v_{j} \nabla G_{j}(y)+\sum_{k \in K_{0}} w_{k} \nabla H_{k}(y)\right]\right.\right. \\
- & {\left.\left.\left[N(y, u)+\Lambda_{0}(y, v, w)\right] \nabla g_{i}(y)\right\}\right)+\frac{1}{2}\left\langle z, \sum_{i=1}^{p} u_{i}\left\{D ( y , u ) \left[\nabla^{2} f_{i}(y)\right.\right.\right.} \\
& \left.\left.\left.+\sum_{j \in J_{0}} v_{j} \nabla^{2} G_{j}(y)+\sum_{k \in K_{0}} w_{k} \nabla^{2} H_{k}(y)\right]-\left[N(y, u)+\Lambda_{0}(y, v, w)\right] \nabla^{2} g_{i}(y)\right\} z\right\rangle \\
> & \sum_{t=1}^{M} \tilde{\rho}_{t}(x, y)\|\theta(x, y)\|^{m} \geq-\bar{\rho}(x, y)\|\theta(x, y)\|^{m},
\end{aligned}
$$

which contradicts (2.12). Therefore, we conclude that $\bar{\phi}(x) \geq \psi_{I}(y, z, u, v, w)$.

(d) The proof is similar to that of part (c).

Theorem 2.3 (Strong duality) Let $x^{*}$ be a normal optimal solution of $(P)$ and assume that any one of the four sets of conditions specified in Theorem 2.2 is satisfied for all feasible solutions of (DI). Then, for each $z^{*} \in C\left(x^{*}\right)$, there exist $u^{*} \in U, v^{*} \in \mathbb{R}_{+}^{q}$, and $w^{*} \in \mathbb{R}^{r}$, such that $\mathcal{S}^{*} \equiv$ $\left(x^{*}, z^{*}, u^{*}, v^{*}, w^{*}\right)$ is an optimal solution of (DI) and $\varphi\left(x^{*}\right)=\psi_{I}\left(\mathcal{S}^{*}\right)$.
Proof Since $x^{*}$ is a normal optimal solution of $(P)$, by Theorem 2.1, for each $z^{*} \in C\left(x^{*}\right)$, there exist $u^{*} \in U, \bar{v} \in \mathbb{R}_{+}^{q}$, and $\bar{w} \in \mathbb{R}^{r}$, such that

$$
\begin{gathered}
\sum_{i=1}^{p} u_{i}^{*}\left[D\left(x^{*}, u^{*}\right) \nabla f_{i}\left(x^{*}\right)-N\left(x^{*}, u^{*}\right) \nabla g_{i}\left(x^{*}\right)\right] \\
+\sum_{j=1}^{q} \bar{v}_{j} \nabla G_{j}\left(x^{*}\right)+\sum_{k=1}^{r} \bar{w}_{k} \nabla H_{k}\left(x^{*}\right)=0
\end{gathered}
$$

$$
\begin{aligned}
& \left\langle z^{*},\left\{\sum_{i=1}^{p} u_{i}^{*}\left[D\left(x^{*}, u^{*}\right) \nabla^{2} f_{i}\left(x^{*}\right)-N\left(x^{*}, u^{*}\right) \nabla^{2} g_{i}\left(x^{*}\right)\right]\right.\right. \\
& \left.\left.\quad+\sum_{j=1}^{q} \bar{v}_{j} \nabla^{2} G_{j}\left(x^{*}\right)+\sum_{k=1}^{r} \bar{w}_{k} \nabla^{2} H_{k}\left(x^{*}\right)\right\} z^{*}\right\rangle \geq 0 \\
& \max _{1 \leq i \leq p} \frac{f_{i}\left(x^{*}\right)}{g_{i}\left(x^{*}\right)}=\frac{N\left(x^{*}, u^{*}\right)}{D\left(x^{*}, u^{*}\right)} \\
& \bar{v}_{j} G_{j}\left(x^{*}\right)=0, j \in \underline{q} .
\end{aligned}
$$

Now, choosing $v_{j}^{*}=\bar{v}_{j} / D\left(x^{*}, u^{*}\right)$ for each $j \in J_{0}, v_{j}^{*}=\bar{v}_{j}$ for each $j \in \underline{q} \backslash J_{0}, w_{k}^{*}=\bar{w}_{k} / D\left(x^{*}, u^{*}\right)$ for each $k \in K_{0}$, and $w_{k}^{*}=\bar{w}_{k}$ for each $k \in \underline{r} \backslash K_{0}$, and noticing that $x^{*} \in \mathbb{F}$, we deduce the following relations from (2.13) to (2.16):

$$
\begin{aligned}
& \sum_{i=1}^{p} u_{i}^{*}\left\{D\left(x^{*}, u^{*}\right)\left[\nabla f_{i}\left(x^{*}\right)+\sum_{j \in J_{0}} v_{j}^{*} \nabla G_{j}\left(x^{*}\right)+\sum_{k \in K_{0}} w_{k}^{*} \nabla H_{k}\left(x^{*}\right)\right]\right. \\
& \left.-\left[N\left(x^{*}, u^{*}\right)+\Lambda_{0}\left(x^{*}, v^{*}, w^{*}\right)\right] \nabla g_{i}\left(x^{*}\right)\right\}+\sum_{j \in q} v_{J}^{*} \nabla G_{j}\left(x^{*}\right) \\
& +\sum_{k \in r \backslash K_{0}} w_{k}^{*} \nabla H_{k}\left(x^{*}\right)=0, \\
& \left\langle z^{*}, \sum_{i=1}^{p} u_{i}^{*}\left\{D\left(x^{*}, u^{*}\right)\left[\nabla^{2} f_{i}\left(x^{*}\right)+\sum_{j \in J_{0}} v_{j}^{*} \nabla^{2} G_{j}\left(x^{*}\right)+\sum_{k \in K_{0}} w_{k}^{*} \nabla^{2} H_{k}\left(x^{*}\right)\right]\right.\right. \\
& \left.-\left[N\left(x^{*}, u^{*}\right)+\Lambda_{0}\left(x^{*}, v^{*}, w^{*}\right)\right] \nabla^{2} g_{i}\left(x^{*}\right)\right\}+\sum_{j \in q \backslash J_{0}} v_{j}^{*} \nabla^{2} G_{j}\left(x^{*}\right) \\
& \left.\left.+\sum_{k \in r \backslash \backslash K_{0}} w_{k}^{*} \nabla^{2} H_{k}\left(x^{*}\right)\right\} z^{*}\right\rangle \geq 0, \\
& \sum_{j \in J_{t}} v_{j}^{*} G_{j}\left(x^{*}\right)+\sum_{k \in K_{t}} w_{k}^{*} H_{k}\left(x^{*}\right)=0, t \in \underline{M} \cup\{0\}, \\
& \varphi\left(x^{*}\right)=\frac{N\left(x^{*}, u^{*}\right)+\Lambda_{0}\left(x^{*}, v^{*}, w^{*}\right)}{D\left(x^{*}, u^{*}\right)} .
\end{aligned}
$$

From (2.17) to (2.19), it is clear that $\mathcal{S}^{*}$ is a feasible solution of $(D I)$, and from (2.20), we see that $\varphi\left(x^{*}\right)=\psi_{I}\left(\mathcal{S}^{*}\right)$. If $\mathcal{S}^{*}$ were not an optimal solution of $(D I)$, then there would exist a feasible solution $\mathcal{S}^{\circ} \equiv$ $\left(x^{\circ}, z^{\circ}, u^{\circ}, v^{\circ}, w^{\circ}\right) \quad$ of $\quad(D I), \quad$ such that $\psi_{I}\left(\mathcal{S}^{\circ}\right)>\psi_{I}\left(\mathcal{S}^{*}\right)=\varphi\left(x^{*}\right)$, which contradicts Theorem 2.2. Therefore, we conclude that $\mathcal{S}^{*}$ is an optimal solution of (DI). 
Theorem 2.4 (Strict Converse Duality) Let $x^{*}$ be $a$ normal optimal solution of $(P)$, let $\tilde{\mathcal{S}} \equiv(\tilde{x}, \tilde{z}, \tilde{u}, \tilde{v}, \tilde{w})$ be an optimal solution of $(D I)$, and assume that any one of the following four sets of conditions holds:

(a) The assumptions specified in part (a) of Theorem 2.1 are satisfied for the feasible solution $\tilde{\mathcal{S}}$ of (DI), $\bar{\phi}(a)>0 \Rightarrow a>0$, and the function $\xi \rightarrow$ $\Phi(\xi, \tilde{x}, \tilde{u}, \tilde{v}, \tilde{w}) \quad$ is strictly $\quad(\mathcal{F}, \beta, \bar{\phi}, \bar{\rho}, \theta, m)$-pseudosounivex at $\tilde{x}$.

(b) The assumptions specified in part (b) of Theorem 2.1 are satisfied for the feasible solution $\tilde{\mathcal{S}}$ of (DI), $\bar{\phi}(a)>0 \Rightarrow a>0$, and the function $\xi \rightarrow$ $\Phi(\xi, \tilde{x}, \tilde{u}, \tilde{v}, \tilde{w})$ is $(\mathcal{F}, \beta, \bar{\phi}, \bar{\rho}, \theta, m)$-quasisounivex at $\tilde{x}$.

(c) The assumptions specified in part (c) of Theorem 2.1 are satisfied for the feasible solution $\tilde{\mathcal{S}}$ of $(D I)$, $\bar{\phi}(a)>0 \Rightarrow a>0$, and the function $\xi \rightarrow$ $\Phi(\xi, \tilde{x}, \tilde{u}, \tilde{v}, \tilde{w})$ is $(\mathcal{F}, \beta, \bar{\phi}, \bar{\rho}, \theta, m)$-quasisounivex at $\tilde{x}$.

(d) The assumptions specified in part (d) of Theorem 2.1 are satisfied for the feasible solution $\tilde{\mathcal{S}}$ of $(D I)$, $\bar{\phi}(a)>0 \Rightarrow a>0$, and the function $\xi \rightarrow$ $\Phi(\xi, \tilde{x}, \tilde{u}, \tilde{v}, \tilde{w})$ is $(\mathcal{F}, \beta, \bar{\phi}, \bar{\rho}, \theta, m)$-quasisounivex at $\tilde{x}$.

Then, $\tilde{x}=x^{*}$ and $\varphi\left(x^{*}\right)=\psi_{I}(\tilde{\mathcal{S}})$.

Proof Since $x^{*}$ is a normal optimal solution of $(P)$, by Theorem 2.3, there exist $z^{*} \in C\left(x^{*}\right), u^{*}, v^{*}$, and $w^{*}$, such that $\mathcal{S}^{*} \equiv\left(x^{*}, z^{*}, u^{*}, v^{*}, w^{*}\right)$ is a feasible solution of $(D I)$ and $\varphi\left(x^{*}\right)=\psi_{I}\left(\mathcal{S}^{*}\right)$. (a): Suppose to the contrary that $\tilde{x} \neq x^{*}$. Now, proceeding as in the proof of part (a) of Theorem 2.2 (with $x$ replaced by $x^{*}$ and $\mathcal{S}$ by $\tilde{\mathcal{S}}$ ), we arrive at the strict inequality

$\sum_{i=1}^{p} \tilde{u}_{i}\left\{D(\tilde{x}, \tilde{u}) f_{i}(x)-\left[N(\tilde{x}, \tilde{u})+\Lambda_{0}(\tilde{x}, \tilde{v}, \tilde{w})\right] g_{i}(x)\right\}>0$.

Using this inequality along with Lemma 2.1 , as in the proof of Theorem 2.2, we get $\varphi\left(x^{*}\right)>\psi_{I}(\tilde{\mathcal{S}})$, which contradicts the fact that $\varphi\left(x^{*}\right)=\psi_{I}\left(\mathcal{S}^{*}\right) \leq \psi_{I}(\tilde{\mathcal{S}})$. (b)-(d): The proofs are similar to that of part (a).

As pointed out earlier, the duality models $(D I)$ and $(\tilde{D} I)$ are two families of dual problems whose members can easily be identified by appropriate choices of the partitioning sets $J_{0}, J_{1}, \ldots, J_{M}, K_{0}, K_{1}, \ldots, K_{M}$. To illustrate this possibility, we shall next briefly discuss some special cases of (DI) and $(\tilde{D} I)$.

If we choose $J_{0}=\underline{q}$ and $K_{0}=\underline{r}$ in (DI) and $(\tilde{D} I)$, then we obtain the following dual problems for $(P)$ :
(DIa) Maximize

$\frac{\sum_{i=1}^{p} u_{i} f_{i}(y)+\sum_{j=1}^{q} v_{j} G_{j}(y)+\sum_{k=1}^{r} w_{k} H_{k}(y)}{\sum_{i=1}^{p} u_{i} g_{i}(y)}$

subject to

$$
\begin{gathered}
D(y, u)\left[\sum_{i=1}^{p} u_{i} \nabla f_{i}(y)+\sum_{j=1}^{q} v_{j} \nabla G_{j}(y)+\sum_{k=1}^{r} w_{k} \nabla H_{k}(y)\right] \\
-[N(y, u)+\Lambda(y, v, w)] \sum_{i=1}^{p} u_{i} \nabla g_{i}(y)=0, \\
\left\langle z,\left\{D(y, u)\left[\sum_{i=1}^{p} u_{i} \nabla^{2} f_{i}(y)+\sum_{j=1}^{q} v_{j} \nabla^{2} G_{j}(y)+\sum_{k=1}^{r} w_{k} \nabla^{2} H_{k}(y)\right]\right.\right. \\
\left.\left.\left.-[N(y, u)+\Lambda(y, v, w)] \sum_{i=1}^{p} u_{i} \nabla^{2} g_{i}(y)\right]\right\} z\right\rangle \geq 0,
\end{gathered}
$$

$y \in X, z \in C(y), u \in U, v \in \mathbb{R}_{+}^{q}, w \in \mathbb{R}^{r}$,

where

$$
\Lambda(y, v, w)=\sum_{j=1}^{q} v_{j} G_{j}(y)+\sum_{k=1}^{r} w_{k} H_{k}(y) ;
$$

(D̃Ia) Maximize

$\frac{\sum_{i=1}^{p} u_{i} f_{i}(y)+\sum_{j=1}^{q} v_{j} G_{j}(y)+\sum_{k=1}^{r} w_{k} H_{k}(y)}{\sum_{i=1}^{p} u_{i} g_{i}(y)}$

subject to

$$
\begin{array}{r}
\mathcal{F}\left(x, y ; D(y, u)\left[\sum_{i=1}^{p} u_{i} \nabla f_{i}(y)+\sum_{j=1}^{q} v_{j} \nabla G_{j}(y)+\sum_{k=1}^{r} w_{k} \nabla H_{k}(y)\right]\right. \\
\left.-[N(y, u)+\Lambda(y, v, w)] \sum_{i=1}^{p} u_{i} \nabla g_{i}(y)\right) \geq 0 \text { for all } x \in \mathbb{F}, \\
\left\langle z,\left\{D(y, u)\left[\sum_{i=1}^{p} u_{i} \nabla^{2} f_{i}(y)+\sum_{j=1}^{q} v_{j} \nabla^{2} G_{j}(y)+\sum_{k=1}^{r} w_{k} \nabla^{2} H_{k}(y)\right]\right.\right. \\
\left.\left.\left.-[N(y, u)+\Lambda(y, v, w)] \sum_{i=1}^{p} u_{i} \nabla^{2} g_{i}(y)\right]\right\} z\right\rangle \geq 0, \\
y \in X, z \in C(y), u \in U, v \in \mathbb{R}_{+}^{q}, w \in \mathbb{R}^{r},
\end{array}
$$

where $\mathcal{F}(x, y ; \cdot)$ is a sublinear function from $\mathbb{R}^{n}$ to $\mathbb{R}$.

If we choose $M=q+r, J_{0}=\emptyset, K_{0}=\emptyset, J_{t}=\{t\}$, $K_{t}=\emptyset, t \in \underline{q}$, and $J_{t}=\emptyset, K_{t}=\{t\}, t \in \underline{r}$, then $(D I)$ and $(\tilde{D} I)$ reduce to the following dual problems for $(P)$ :

(DIb) Maximize $\frac{\sum_{i=1}^{p} u_{i} f_{i}(y)}{\sum_{i=1}^{p} u_{i} g_{i}(y)}$

subject to

$$
\begin{aligned}
\sum_{i=1}^{p} u_{i}\left[D(y, u) \nabla f_{i}(y)\right. & \left.-N(y, u) \nabla g_{i}(y)\right]+\sum_{j=1}^{q} v_{j} \nabla G_{j}(y) \\
+ & \sum_{k=1}^{r} w_{k} \nabla H_{k}(y)=0,
\end{aligned}
$$




$$
\begin{aligned}
\langle z, & \left\{\sum_{i=1}^{p} u_{i}\left[D(y, u) \nabla^{2} f_{i}(y)-N(y, u) \nabla^{2} g_{i}(y)\right]+\sum_{j=1}^{q} v_{j} \nabla^{2} G_{j}(y)\right. \\
& \left.\left.+\sum_{k=1}^{r} w_{k} \nabla^{2} H_{k}(y)\right\} z\right\rangle \geq 0,
\end{aligned}
$$

$v_{j} G_{j}(y) \geq 0, j \in \underline{q}$,

$w_{k} H_{k}(y) \geq 0, k \in \underline{r}$,

$y \in X, z \in C(y), u \in U, v \in \mathbb{R}_{+}^{q}, w \in \mathbb{R}^{r} ;$

$(\tilde{D} I b)$ Maximize
subject to $\frac{\sum_{i=1}^{p} u_{i} f_{i}(y)}{\sum_{i=1}^{p} u_{i} g_{i}(y)}$

$$
\begin{array}{r}
\mathcal{F}\left(x, y ; D(y, u)\left[\sum_{i=1}^{p} u_{i} \nabla f_{i}(y)+\sum_{j=1}^{q} v_{j} \nabla G_{j}(y)+\sum_{k=1}^{r} w_{k} \nabla H_{k}(y)\right]\right. \\
\left.-[N(y, u)+\Lambda(y, v, w)] \sum_{i=1}^{p} u_{i} \nabla g_{i}(y)\right) \geq 0 \text { for all } x \in \mathbb{F},
\end{array}
$$

$\left\langle z,\left\{\sum_{i=1}^{p} u_{i}\left[D(y, u) \nabla^{2} f_{i}(y)-N(y, u) \nabla^{2} g_{i}(y)\right]+\sum_{j=1}^{q} v_{j} \nabla^{2} G_{j}(y)\right.\right.$

$\left.\left.+\sum_{k=1}^{r} w_{k} \nabla^{2} H_{k}(y)\right\} z\right\rangle \geq 0$

$v_{j} G_{j}(y) \geq 0, j \in \underline{q}$,

$w_{k} H_{k}(y) \geq 0, k \in \underline{r}$,

$y \in X, z \in C(y), u \in U, v \in \mathbb{R}_{+}^{q}, w \in \mathbb{R}^{r}$.

In a similar manner, we can identify many other special cases of $(D I)$ and $(\tilde{D} I)$. Evidently, Theorems 2.1-2.3 can be specialized for $(D I a),(\tilde{D} I a),(D I b)$, and $(\tilde{D} I b)$ in a straightforward fashion.

The dual problems $(D I a),(\tilde{D} I a),(D I b)$, and $(\tilde{D} I b)$ were investigated previously in [10] with $\mathcal{F}\left(x, x^{*} ; \nabla f\left(x^{*}\right)\right)=$ $\left\langle\nabla f\left(x^{*}\right), \eta\left(x, x^{*}\right)\right\rangle$, where $\eta$ is a function from $X \times X$ to $\mathbb{R}^{n}$, and a great variety of duality results were established under various (strict) $(\phi, \eta, \rho, \theta, m)$-sonvexity, (strict) $(\phi, \eta, \rho$, $\theta, m)$-pseudosonvexity, and (prestrict) $(\phi, \eta, \rho, \theta, m)$-quasisonvexity hypotheses.

\section{Duality model II and duality theorems}

In Theorems 2.2-2.4, various generalized $(\mathcal{F}, \beta, \phi$, $\rho, \theta, m)$-sounivexity conditions were imposed on the function $\xi \rightarrow \Phi(\xi, y, u, v, w)$, which is the weighted sum of the functions

$$
\begin{aligned}
\Phi_{i}(\xi, y, v, w)= & D(y, u)\left[f_{i}(\xi)+\sum_{j \in J_{0}} v_{j} G_{j}(\xi)+\sum_{k \in K_{0}} w_{k} H_{k}(\xi)\right] \\
& -\left[N(y, u)+\Lambda_{0}(y, v, w)\right] g_{i}(\xi), i \in \underline{p} .
\end{aligned}
$$

In this section, we consider some generalized versions of $(D I)$ and $(\tilde{D} I)$, and prove weak and strong duality theorems in which we assume that the individual functions $\xi \rightarrow \Phi_{i}(\xi, y, v, w), i \in \underline{p}$, satisfy appropriate generalized $(\mathcal{F}, \beta, \phi, \rho, \theta, m)$-sounivexity hypotheses. This can be accomplished by appending an additional system of inequality constraints to $(D I)$ and $(\tilde{D} I)$.

Consider the following two problems:

(DII)

$\frac{\sum_{i=1}^{p} u_{i} f_{i}(y)+\sum_{j \in J_{0}} v_{j} G_{j}(y)+\sum_{k \in K_{0}} w_{k} H_{k}(y)}{\sum_{i=1}^{p} u_{i} g_{i}(y)}$

Maximize

subject to

$$
\begin{aligned}
& \sum_{i=1}^{p} u_{i}\left\{D(y, u)\left[\nabla f_{i}(y)+\sum_{j \in J_{0}} v_{j} \nabla G_{j}(y)+\sum_{k \in K_{0}} w_{k} \nabla H_{k}(y)\right]\right. \\
& \left.\quad-\left[N(y, u)+\Lambda_{0}(y, v, w)\right] \nabla g_{i}(y)\right\}+\sum_{j \in \underline{q} \backslash J_{0}} v_{j} \nabla G_{j}(y) \\
& \quad+\sum_{k \in \underline{r} \backslash K_{0}} w_{k} \nabla H_{k}(y)=0 \\
& \left.\quad-\left[N(y, u)+\Lambda_{0}(y, v, w)\right] \nabla^{2} g_{i}(y)\right\}+\sum_{j \in q} v_{j} \nabla^{2} G_{j}(y) \\
& \left.\left.\quad+\sum_{k \in \underline{r} \backslash K_{0}} w_{k} \nabla^{2} H_{k}(y)\right\} z\right\rangle \geq 0, \\
& D(y, u)\left[f_{i}(y)+\sum_{j \in J_{0}} v_{j} G_{j}(y)+\sum_{k \in K_{0}} w_{k} H_{k}(y)\right] \\
& \quad-\left[N(y, u)+\Lambda_{0}(y, v, w)\right] g_{i}(y) \geq 0, i \in \underline{p}, \\
& \sum \sum_{j \in J_{t}} v_{j} G_{j}(y)+\sum_{k \in K_{t}} w_{k} H_{k}(y) \geq 0, t \in \underline{M}, \\
& y \in \in, z \in C(y), u \in U, v \in \mathbb{R}_{+}^{q}, w \in \mathbb{R}^{r}
\end{aligned}
$$

$(\tilde{D} I I)$

$\frac{\sum_{i=1}^{p} u_{i} f_{i}(y)+\sum_{j \in J_{0}} v_{j} G_{j}(y)+\sum_{k \in K_{0}} w_{k} H_{k}(y)}{\sum_{i=1}^{p} u_{i} g_{i}(y)}$

Maximize

subject to (3.2)-(3.5) and

$$
\begin{aligned}
\mathcal{F}(x, y ; & \sum_{i=1}^{p} u_{i}\left\{D(y, u)\left[\nabla f_{i}(y)+\sum_{j \in J_{0}} v_{j} \nabla G_{j}(y)+\sum_{k \in K_{0}} w_{k} \nabla H_{k}(y)\right]\right. \\
- & {\left.\left.\left[N(y, u)+\Lambda_{0}(y, v, w)\right] \nabla g_{i}(y)\right\}+\sum_{j \in q} v_{j} \nabla G_{j}(y)+\sum_{k \in \underline{r} \backslash K_{0}} w_{k} \nabla H_{k}(y)\right) }
\end{aligned}
$$

$\geq 0$ for all $x \in \mathbb{F}$,

where $\mathcal{F}(x, y ; \cdot)$ is a sublinear function from $\mathbb{R}^{n}$ to $\mathbb{R}$.

The comments and observations made earlier about the relationship between $(D I)$ and $(\tilde{D} I)$ are, of course, also valid for $(D I I)$ and $(\tilde{D} I I)$.

The following two theorems show that $(D I I)$ is a dual problem for $(P)$. 
Theorem 3.1 (Weak duality) Let $x$ and $\mathcal{S} \equiv(y, z, u, v, w)$ be arbitrary feasible solutions of $(P)$ and $(D I I)$, respectively, and assume that any one of the following seven sets of hypotheses is satisfied:

(i) $\quad$ For each $\quad i \in I_{+} \equiv\left\{i \in \underline{p}: u_{i}>0\right\}, \xi \rightarrow$ $\Phi_{i}(\xi, y, v, w) \quad$ is $\quad\left(\mathcal{F}, \beta, \bar{\phi}_{i}, \bar{\rho}_{i}, \theta, m\right)$-pseudosounivex at $y, \bar{\phi}_{i}$ is strictly increasing, and $\bar{\phi}_{i}(0)=0$

(ii) For each $t \in \underline{M}, \xi \rightarrow \Lambda_{t}(\xi, v, w) \quad$ is $\left(\mathcal{F}, \beta, \tilde{\phi}_{t}, \tilde{\rho}_{t}, \theta, m\right)$-quasisounivex at $y, \tilde{\phi}_{t}$ is increasing, and $\tilde{\phi}_{t}(0)=0$;

(iii) $\quad \sum_{i \in I_{+}} u_{i} \bar{\rho}_{i}(x, y)+\sum_{t=1}^{M} \tilde{\rho}_{t}(x, y) \geq 0$;

(b)

(i) For each $i \in I_{+}, \xi \rightarrow \Phi_{i}(\xi, y, v, w)$ is prestrictly $\left(\mathcal{F}, \beta, \bar{\phi}_{i}, \bar{\rho}_{i}, \theta, m\right)$-quasisounivex at $y, \bar{\phi}_{i}$ is strictly increasing, and $\bar{\phi}_{i}(0)=0$;

(ii) For each $t \in \underline{m}, \xi \rightarrow \Lambda_{t}(\xi, v, w)$ is strictly $\left(\mathcal{F}, \beta, \tilde{\phi}_{t}, \tilde{\rho}_{t}, \theta, m\right)$-pseudosounivex at $y, \tilde{\phi}_{t}$ is increasing, and $\tilde{\phi}_{t}(0)=0$;

(iii) $\quad \sum_{i \in I_{+}} u_{i} \bar{\rho}_{i}(x, y)+\sum_{t=1}^{M} \tilde{\rho}_{t}(x, y) \geq 0$;

(c)

(i) For each $i \in I_{+}, \xi \rightarrow \Phi_{i}(\xi, y, v, w)$ is prestrictly $\left(\mathcal{F}, \beta, \bar{\phi}_{i}, \bar{\rho}_{i}, \theta, m\right)$-quasisounivex at $y, \bar{\phi}_{i}$ is strictly increasing, and $\bar{\phi}_{i}(0)=0$;

(ii) For each $t \in \underline{M}, \xi \rightarrow \Lambda_{t}(\xi, v, w)$ is $\left(\mathcal{F}, \beta, \tilde{\phi}_{t}, \tilde{\rho}_{t}, \theta, m\right)$-quasisounivex at $y, \tilde{\phi}_{t}$ is increasing, and $\tilde{\phi}_{t}(0)=0$;

(iii) $\quad \sum_{i \in I_{+}} u_{i} \bar{\rho}_{i}(x, y)+\sum_{t=1}^{M} \tilde{\rho}_{t}(x, y)>0$;

(d)

(i) For each $\quad i \in I_{1+}, \xi \rightarrow \Phi_{i}(\xi, y, v, w) \quad$ is $\left(\mathcal{F}, \beta, \bar{\phi}_{i}, \bar{\rho}_{i}, \theta, m\right)$-pseudosounivex at $y$, for each $i \in I_{2+}, \xi \rightarrow \Phi_{i}(\xi, y, v, w)$ is prestrictly $\left(\mathcal{F}, \beta, \bar{\phi}_{i}, \bar{\rho}_{i}, \theta, m\right)$-quasisounivex at $y$, and for each $i \in I_{+}, \bar{\phi}_{i}$ is strictly increasing and $\bar{\phi}_{i}(0)=0$, where $\left\{I_{1+}, I_{2+}\right\}$ is a partition of $I_{+}$;

(ii) For each $t \in \underline{M}, \xi \rightarrow \Lambda_{t}(\xi, v, w)$ is strictly $\left(\mathcal{F}, \beta, \tilde{\phi}_{t}, \tilde{\rho}_{t}, \theta, m\right)$-pseudosounivex at $y, \tilde{\phi}_{t}$ is increasing, and $\tilde{\phi}_{t}(0)=0$;

(iii) $\quad \sum_{i \in I_{+}} u_{i} \bar{\rho}_{i}(x, y)+\sum_{t=1}^{M} \tilde{\rho}_{t}(x, y) \geq 0$;

(e)

(i) For each $i \in I_{1+} \neq \emptyset, \xi \rightarrow \Phi_{i}(\xi, y, v, w)$ is $\left(\mathcal{F}, \beta, \bar{\phi}_{i}, \bar{\rho}_{i}, \theta, m\right)$-pseudosounivex at $y$, for each $i \in I_{2+}, \xi \rightarrow \Phi_{i}(\xi, y, v, w)$ is prestrictly $\left(\mathcal{F}, \beta, \bar{\phi}_{i}, \bar{\rho}_{i}, \theta\right)$-quasisounivex at $y$, and for each $i \in I_{+}, \bar{\phi}_{i}$ is strictly increasing and $\bar{\phi}_{i}(0)=0$, where $\left\{I_{1+}, I_{2+}\right\}$ is a partition of $I_{+}$;

(ii) For each $t \in \underline{M}, \xi \rightarrow \Lambda_{t}(\xi, v, w) \quad$ is $\left(\mathcal{F}, \beta, \tilde{\phi}_{t}, \tilde{\rho}_{t}, \theta, m\right)$-quasisounivex at $y, \tilde{\phi}_{t}$ is increasing, and $\tilde{\phi}_{t}(0)=0$;

(iii) $\sum_{i \in I_{+}} u_{i} \bar{\rho}_{i}(x, y)+\sum_{t=1}^{M} \tilde{\rho}_{t}(x, y) \geq 0$;

(i) For each $i \in I_{+}, \xi \rightarrow \Phi_{i}(\xi, y, v, w)$ is prestrictly $\left(\mathcal{F}, \beta, \bar{\phi}_{i}, \bar{\rho}_{i}, \theta\right)$-quasisounivex at $y, \bar{\phi}_{i}$ is strictly increasing, and $\bar{\phi}_{i}(0)=0$;

(ii) For each $t \in \underline{M_{1}} \neq \emptyset, \xi \rightarrow \Lambda_{t}(\xi, v, w)$ is strictly $\left(\mathcal{F}, \beta, \tilde{\phi}_{t}, \tilde{\rho}_{t}, \theta, m\right)$-pseudosounivex at $y$, for each $t \in \underline{M_{2}}, \xi \rightarrow \Lambda_{t}(\xi, v, w)$ is $\left(\mathcal{F}, \beta, \tilde{\phi}_{t}, \tilde{\rho}_{t}, \theta, m\right)$-quasisounivex at $y$, and for each $t \in \underline{M}, \tilde{\phi}_{t}$ is increasing and $\tilde{\phi}_{t}(0)=0$, where $\left\{\underline{M_{1}}, \underline{M_{2}}\right\}$ is a partition of $\underline{M}$

(iii) $\sum_{i \in I_{+}} u_{i} \bar{\rho}_{i}(x, y)+\sum_{t=1}^{M} \tilde{\rho}_{t} \geq 0$;

(g)

(i) For each $\quad i \in I_{1+}, \xi \rightarrow \Phi_{i}(\xi, y, v, w) \quad$ is $\left(\mathcal{F}, \beta, \bar{\phi}_{i}, \bar{\rho}_{i}, \theta, m\right)$-pseudosounivex at $y$, for each $i \in I_{2+}, \xi \rightarrow \Phi_{i}(\xi, y, v, w)$ is prestrictly $\left(\mathcal{F}, \beta, \bar{\phi}_{i}, \bar{\rho}_{i}, \theta, m\right)$-quasisounivex at $y$, and for each $i \in I_{+}, \bar{\phi}_{i}$ is strictly increasing and $\bar{\phi}_{i}(0)=0$, where $\left\{I_{1+}, I_{2+}\right\}$ is a partition of $I_{+}$;

(ii) For each $t \in \underline{M_{1}}, \xi \rightarrow \Lambda_{t}(\xi, v, w)$ is strictly $\left(\mathcal{F}, \beta, \tilde{\phi}_{t}, \tilde{\rho}_{t}, \theta, m\right)$-pseudosounivex at $y$,for each $\quad t \in M_{2}, \xi \rightarrow \Lambda_{t}(\xi, v, w) \quad$ is $\left(\mathcal{F}, \beta, \tilde{\phi}_{t}, \tilde{\rho}_{t}, \theta, m\right)$-quasisounivex at $y$, and for $t \in \underline{M}, \tilde{\phi}_{t}$ is increasing and $\tilde{\phi}_{t}(0)=0$, where $\left\{M_{1}, M_{2}\right\}$ is a partition of $\underline{M}$;

(iii) $\sum_{i \in I_{+}} u_{i} \bar{\rho}_{i}(x, y)+\sum_{t=1}^{M} \tilde{\rho}_{t}(x, y) \geq 0$;

(iv) $\quad I_{1+} \neq \emptyset, \quad \underline{M_{1}} \neq \emptyset, \quad$ or $\quad \sum_{i \in I_{+}} u_{i} \bar{\rho}_{i}(x, y)+$ $\sum_{t=1}^{M} \tilde{\rho}_{t}(x, y)>0$.

Then, $\varphi(x) \geq \psi_{I I}(\mathcal{S})$, where $\psi_{I I}$ is the objective function of (DII).

Proof (a): Suppose to the contrary that $\varphi(x)<\psi_{I I}(\mathcal{S})$. This implies that

$D(y, u) f_{i}(x)-\left[N(y, u)+\Lambda_{0}(y, v, w)\right] g_{i}(x)<0, i \in \underline{p}$.

Keeping in mind that $v \geq 0$, we see that for each $i \in I_{+}$, 


$$
\begin{aligned}
\Phi_{i}(x, y, v, w) & =D(y, u)\left[f_{i}(x)+\sum_{j \in J_{0}} v_{j} G_{j}(x)+\sum_{k \in K_{0}} w_{k} H_{k}(x)\right] \\
& -\left[N(y, u)+\Lambda_{0}(y, u, v)\right] g_{i}(x) \\
& \leq D(y, u) f_{i}(x)-\left[N(y, u)+\Lambda_{0}(y, v, w)\right] g_{i}(x)
\end{aligned}
$$

(by the primal feasibility of $x$ )

$$
<0 \text { (by (3.6)) } \leq \Phi_{i}(y, y, v, w) \text { (by (3.3)) , }
$$

and so it follows from the properties of $\bar{\phi}_{i}$ that

$$
\bar{\phi}_{i}\left(\Phi_{i}(x, y, v, w)-\Phi_{i}(y, y, v, w)\right)<0,
$$

which in view of (i) implies that

$$
\begin{aligned}
\mathcal{F} & \left(x, y ; \beta(x, y)\left\{D(y, u)\left[\nabla f_{i}(y)+\sum_{j \in J_{0}} u_{j} \nabla G_{j}(y)+\sum_{k \in K_{0}} w_{k} \nabla H_{k}(y)\right]\right.\right. \\
& \left.\left.-\left[N(y, u)+\Lambda_{0}(y, u, v)\right] \nabla g_{i}(x)\right\}\right)+\frac{1}{2}\left\langle z,\left\{D ( y , u ) \left[\nabla^{2} f_{i}(y)\right.\right.\right. \\
& \left.+\sum_{j \in J_{0}} v_{j} \nabla^{2} G_{j}(y)+\sum_{k \in K_{0}} w_{k} \nabla^{2} H_{k}(y)\right] \\
& \left.\left.-\left[N(y, u)+\Lambda_{0}(y, v, w)\right] \nabla^{2} g_{i}(x)\right\} z\right\rangle<-\bar{\rho}_{i}(x, y)\|\theta(x, y)\|^{m} .
\end{aligned}
$$

Since $u \geq 0, u_{i}=0$ for each $i \in \underline{p} \backslash I_{+}, \sum_{i=1}^{p} u_{i}=1$, and $\mathcal{F}(x, y ; \cdot)$ is sublinear, the above inequalities yield

$$
\begin{aligned}
\mathcal{F} & \left(x, y ; \beta(x, y)\left\{\sum _ { i = 1 } ^ { p } u _ { i } \left\{D(y, u)\left[\nabla f_{i}(y)+\sum_{j \in J_{0}} u_{j} \nabla G_{j}(y)+\sum_{k \in K_{0}} w_{k} \nabla H_{k}(y)\right]\right.\right.\right. \\
& \left.\left.\left.-\left[N(y, u)+\Lambda_{0}(y, u, v)\right] \nabla g_{i}(x)\right\}\right\}\right)+\frac{1}{2}\left\langle z,\left\{\sum _ { i = 1 } ^ { p } u _ { i } \left\{D ( y , u ) \left[\nabla^{2} f_{i}(y)\right.\right.\right.\right. \\
& \left.\left.\left.+\sum_{j \in J_{0}} v_{j} \nabla^{2} G_{j}(y)+\sum_{k \in K_{0}} w_{k} \nabla^{2} H_{k}(y)\right]-\left[N(y, u)+\Lambda_{0}(y, u, v)\right] \nabla^{2} g_{i}(x)\right\} z\right\rangle \\
& <-\sum_{i \in I_{+}} u_{i} \bar{\rho}_{i}(x, y)\|\theta(x, y)\|^{m} .
\end{aligned}
$$

As seen in the proof of Theorem 2.2, our assumptions in (ii) lead to

$$
\begin{aligned}
\mathcal{F}\left(x, y ; \beta(x, y) \sum_{t=1}^{M}\left[\sum_{j \in J_{t}} u_{j} \nabla G_{j}(y)+\sum_{k \in K_{t}} w_{k} \nabla H_{k}(y)\right]\right) \\
+\frac{1}{2}\left\langle z,\left[\sum_{j \in J_{t}} u_{j} \nabla^{2} G_{j}(y)+\sum_{k \in K_{t}} w_{k} \nabla^{2} H_{k}(y)\right] z\right\rangle \\
\leq-\sum_{t=1}^{M} \tilde{\rho}_{t}(x, y)\|\theta(x, y)\|^{m},
\end{aligned}
$$

which when combined with (3.6) and (3.7) results in

$$
\begin{aligned}
\mathcal{F}\left(x, y ; \beta(x, y)\left\{\sum _ { i = 1 } ^ { p } u _ { i } \left\{D(y, u)\left[\nabla f_{i}(y)+\sum_{j \in J_{0}} u_{j} \nabla G_{j}(y)+\sum_{k \in K_{0}} w_{k} \nabla H_{k}(y)\right]\right.\right.\right. \\
\left.\left.\left.-\left[N(y, u)+\Lambda_{0}(y, u, v)\right] \nabla g_{i}(x)\right\}\right\}\right)+\frac{1}{2}\left\langle z,\left\{\sum _ { i = 1 } ^ { p } u _ { i } \left\{D ( y , u ) \left[\nabla^{2} f_{i}(y)\right.\right.\right.\right. \\
\left.+\sum_{j \in J_{0}} v_{j} \nabla^{2} G_{j}(y)+\sum_{k \in K_{0}} w_{k} \nabla^{2} H_{k}(y)\right] \\
\left.\left.-\left[N(y, u)+\Lambda_{0}(y, v, w)\right] \nabla^{2} g_{i}(x)\right\} z\right\rangle \geq \sum_{t=1}^{M} \tilde{\rho}_{t}(x, y)\|\theta(x, y)\|^{m} .
\end{aligned}
$$

In view of (iii), this inequality contradicts (3.7). Hence, $\varphi(x) \geq \psi_{I I}(\mathcal{S})$. (b)-(g): The proofs are similar to that of part (a).

Theorem 3.2 (Strong duality) Let $x^{*}$ be a normal optimal solution of $(P)$ and assume that any one of the seven sets of conditions set forth in Theorem 3.1 is satisfied for all feasible solutions of (DII). Then, for each $z^{*} \in C\left(x^{*}\right)$, there exist $u^{*}, v^{*}$, and $w^{*}$, such that $\left(x^{*}, z^{*}, u^{*}, v^{*}, w^{*}\right)$ is an optimal solution of (DII) and $\varphi\left(x^{*}\right)=$ $\psi_{I I}\left(x^{*}, z^{*}, u^{*}, v^{*}, w^{*}\right)$.

Proof The proof is similar to that of Theorem 2.2.

The duality models $(D I I)$ and $(\tilde{D} I I)$ contain numerous special cases that can easily be identified by appropriate choices of the partitioning sets.

\section{Duality model III and duality theorems}

In this section, we discuss two additional duality models for $(P)$. In these duality formulations, we utilize a partition of $p$ in addition to those of $q$ and $\underline{r}$. This partitioning scheme, which is an extended version of the one initially proposed by Mond and Weir [7], was used by Yang [18] for formulating a generalized duality model for a multiobjective fractional programming problem. In our duality theorems, we impose appropriate generalized $(\mathcal{F}, \beta, \phi, \rho, \theta, m)$-sounivexity requirements on certain combinations of the problem functions.

Let $\left\{I_{0}, I_{1}, \ldots, I_{\ell}\right\}$ be a partition of $p$, such that $\mathcal{L}=\{0,1,2, \ldots, \ell\} \subset \mathcal{M}=\{0,1, \ldots, M\}$, and let the realvalued function $\xi \rightarrow \Pi_{t}(\xi, y, u, v, w)$ be defined, for fixed $u, v$, and $w$, on $X$ by

$$
\begin{aligned}
\Pi_{t}(\xi, y, u, v, w)= & \sum_{i \in I_{t}} u_{i}\left[D(y, u) f_{i}(x)\right. \\
& \left.-N(y, u) g_{i}(x)\right]+\sum_{j \in J_{t}} v_{j} G_{j}(x)+\sum_{k \in K_{t}} w_{k} H_{k}(x), t \in \mathcal{M} .
\end{aligned}
$$

Consider the following two problems:

$$
\text { (DIII) Maximize } \frac{\sum_{i=1}^{p} u_{i} f_{i}(y)}{\sum_{i=1}^{p} u_{i} g_{i}(y)}
$$

subject to

$$
\begin{aligned}
& \sum_{i=1}^{p} u_{i}\left[D(y, u) \nabla f_{i}(y)-N(y, u) \nabla g_{i}(y)\right]+\sum_{j=1}^{q} v_{j} \nabla G_{j}(y) \\
& \quad+\sum_{k=1}^{r} w_{k} \nabla H_{k}(y)=0 \\
& \left\langle z,\left\{\sum_{i=1}^{p} u_{i}\left[D(y, u) \nabla^{2} f_{i}(y)-N(y, u) \nabla^{2} g_{i}(y)\right]\right.\right. \\
& \left.\left.\quad+\sum_{j=1}^{q} v_{j} \nabla^{2} G_{j}(y)+\sum_{k=1}^{r} w_{k} \nabla^{2} H_{k}(y)\right\} z\right\rangle \geq 0
\end{aligned}
$$


$\sum_{i \in I_{t}} u_{i}\left[D(y, u) f_{i}(y)-N(y, u) g_{i}(y)\right]+\sum_{j \in J_{t}} v_{j} G_{j}(y)$

$+\sum_{k \in K_{t}} w_{k} H_{k}(y) \geq 0, t \in \mathcal{M}$,

$\sum_{j \in J_{t}} v_{j} G_{j}(y)+\sum_{k \in K_{t}} w_{k} H_{k}(y) \geq 0, t \in \mathcal{L} \backslash \mathcal{M}$,

$y \in X, z \in C(y), u \in U, v \in \mathbb{R}_{+}^{q}, w \in \mathbb{R}^{r}$

$(\tilde{D} I I I) \quad$ Maximize $\frac{\sum_{i=1}^{p} u_{i} f_{i}(y)}{\sum_{i=1}^{p} u_{i} g_{i}(y)}$

subject to (4.2)-(4.5) and

$$
\begin{aligned}
& \mathcal{F}\left(x, y ; \sum_{i=1}^{p} u_{i}\left[D(y, u) \nabla f_{i}(y)-N(y, u) \nabla g_{i}(y)\right]\right. \\
& \left.\quad+\sum_{j=1}^{q} v_{j} \nabla G_{j}(y)+\sum_{k=1}^{r} w_{k} \nabla H_{k}(y)\right) \geq 0 \text { for all } x \in \mathbb{F},
\end{aligned}
$$

(ii) For each $t \in \mathcal{M} \backslash \mathcal{L}, \xi \rightarrow \Lambda_{t}(\xi, v, w)$ is strictly

(iii) $\quad \sum_{t \in \mathcal{M}} \rho_{t}(x, y) \geq 0$ for all $x \in \mathbb{F}$;

where $\mathcal{F}(x, y ; \cdot)$ is a sublinear function from $\mathbb{R}^{n}$ to $\mathbb{R}$.

The comments and observations made earlier about the relationship between $(D I)$ and $(\tilde{D} I)$ are, of course, also valid for $(D I I I)$ and $(\tilde{D} I I I)$.

The following two theorems show that $(D I I I)$ is a dual problem for $(P)$.

Theorem 4.1 (Weak duality) Let $x$ and $\mathcal{S} \equiv(y, z, u, v, w)$ be arbitrary feasible solutions of $(P)$ and (DIII), respectively, and assume that any one of the following seven sets of hypotheses is satisfied:

(a)

(i) for each $t \in \mathcal{L}, \xi \rightarrow \Pi_{t}(\xi, y, u, v, w)$ is strictly $\left(\mathcal{F}, \beta, \phi_{t}, \rho_{t}, \theta, m\right)$-pseudosounivex at $y, \phi_{t}$ is increasing, and $\phi_{t}(0)=0$;

(ii) for each $t \in \mathcal{M} \backslash \mathcal{L}, \xi \rightarrow \Lambda_{t}(\xi, v, w)$ is $\left(\mathcal{F}, \beta, \phi_{t}, \rho_{t}, \theta, m\right)$-quasisounivex at $y, \phi_{t}$ is increasing, and $\phi_{t}(0)=0$;

(iii) $\quad \sum_{t \in \mathcal{M}} \rho_{t}(x, y) \geq 0$ for all $x \in \mathbb{F}$;

(b)

(i) For each $t \in \mathcal{L}, \xi \rightarrow \Pi_{t}(\xi, y, u, v, w)$ is prestrictly $\left(\mathcal{F}, \beta, \phi_{t}, \rho_{t}, \theta, m\right)$-quasisounivex at $y$, $\phi_{t}$ is increasing, and $\phi_{t}(0)=0$;

(ii) For each $t \in \mathcal{M} \backslash \mathcal{L}, \xi \rightarrow \Lambda_{t}(\xi, v, w)$ is strictly $\left(\mathcal{F}, \beta, \phi_{t}, \rho_{t}, \theta, m\right)$-pseudosounivex at $y, \phi_{t}$ is increasing, and $\phi_{t}(0)=0$;

(iii) $\quad \sum_{t \in \mathcal{M}} \rho_{t}(x, y) \geq 0$ for all $x \in \mathbb{F}$;

(c)

(i) For each $t \in \mathcal{L}, \xi \rightarrow \Pi_{t}(\xi, y, u, v, w)$ is prestrictly $\left(\mathcal{F}, \beta, \phi_{t}, \rho_{t}, \theta, m\right)$-quasisounivex at $y$, $\phi_{t}$ is increasing, and $\phi_{t}(0)=0$; (i) For each $t \in \mathcal{L}_{1}, \xi \rightarrow \Pi_{t}(\xi, y, u, v, w)$ is strictly $\left(\mathcal{F}, \beta, \phi_{t}, \rho_{t}, \theta, m\right)$-pseudosounivex at $y$, for each $t \in \mathcal{L}_{2}, \xi \rightarrow \Pi_{t}(\xi, y, u, v, w)$ is prestrictly $\left(\mathcal{F}, \beta, \phi_{t}, \rho_{t}, \theta, m\right)$-quasisounivex at $y$, and for each $t \in \mathcal{L}, \phi_{t}$ is increasing and $\phi_{t}(0)=0$, where $\left\{\mathcal{L}_{1}, \mathcal{L}_{2}\right\}$ is a partition of $\mathcal{L}$; $\left(\mathcal{F}, \beta, \phi_{t}, \rho_{t}, \theta, m\right)$-pseudosounivex at $y, \phi_{t}$ is increasing, and $\phi_{t}(0)=0$;

(e)

For $\quad$ each $\quad t \in \mathcal{M} \backslash \mathcal{L}, \xi \rightarrow \Lambda_{t}(\xi, v, w)$ is $\left(\mathcal{F}, \beta, \phi_{t}, \rho_{t}, \theta, m\right)$-quasisounivex at $y, \phi_{t}$ is increasing, and $\phi_{t}(0)=0$;

(iii) $\quad \sum_{t \in \mathcal{M}} \rho_{t}(x, y)>0$ for all $x \in \mathbb{F}$;

(i) for each $t \in \mathcal{L}_{1} \neq \emptyset, \xi \rightarrow \Pi_{t}(\xi, y, u, v, w)$ is strictly $\left(\mathcal{F}, \beta, \phi_{t}, \rho_{t}, \theta, m\right)$-pseudosounivex at $y$, for each $t \in \mathcal{L}_{2}, \xi \rightarrow \Pi_{t}(\xi, y, u, v, w)$ is prestrictly $\left(\mathcal{F}, \beta, \phi_{t}, \rho_{t}, \theta, m\right)$-quasisounivex at $y$, and for each $t \in \mathcal{L}, \phi_{t}$ is increasing and $\phi_{t}(0)=0$, where $\left\{\mathcal{L}_{1}, \mathcal{L}_{2}\right\}$ is a partition of $\mathcal{L}$;

(ii) For each $t \in \mathcal{M} \backslash \mathcal{L}, \xi \rightarrow \Lambda_{t}(\xi, v, w)$ is $\left(\mathcal{F}, \beta, \phi_{t}, \rho_{t}, \theta, m\right)$-quasisounivex at $y, \phi_{t}$ is increasing, and $\phi_{t}(0)=0$;

(iii) $\quad \sum_{t \in \mathcal{M}} \rho_{t}(x, y) \geq 0$ for all $x \in \mathbb{F}$;

(i) for each $t \in \mathcal{L}, \xi \rightarrow \Pi_{t}(\xi, y, u, v, w)$ is prestrictly $\left(\mathcal{F}, \beta, \phi_{t}, \rho_{t}, \theta, m\right)$-quasisounivex at $y$, $\phi_{t}$ is increasing, and $\phi_{t}(0)=0$;

(ii) for each $t \in(\mathcal{M} \backslash \mathcal{L})_{1} \neq \emptyset, \xi \rightarrow \Lambda_{t}(\xi, v, w)$ is strictly $\left(\mathcal{F}, \beta, \phi_{t}, \rho_{t}, \theta, m\right)$-pseudosounivex at $y$, for each $t \in(\mathcal{M} \backslash \mathcal{L})_{2}, \xi \rightarrow \Lambda_{t}(\xi, v, w)$ is $\left(\mathcal{F}, \beta, \phi_{t}, \rho_{t}, \theta, m\right)$-quasisounivex at $y$, and for each $t \in \mathcal{L}, \phi_{t}$ is increasing and $\phi_{t}(0)=0$, where $\left\{(\mathcal{M} \backslash \mathcal{L})_{1},(\mathcal{M} \backslash \mathcal{L})_{2}\right\}$ is a partition of $\mathcal{M} \backslash \mathcal{L})$

(iii) $\quad \sum_{t \in \mathcal{M}} \rho_{t}(x, y) \geq 0$ for all $x \in \mathbb{F}$;

$(\mathrm{g})$

(i) for each $t \in \mathcal{L}_{1}, \xi \rightarrow \Pi_{t}(\xi, y, u, v, w)$ is $\left(\mathcal{F}, \beta, \phi_{t}, \rho_{t}, \theta, m\right)$-pseudosounivex at $y$, for each $t \in \mathcal{L}_{2}, \xi \rightarrow \Pi_{t}(\xi, y, u, v, w)$ is prestrictly $\left(\mathcal{F}, \beta, \phi_{t}, \rho_{t}, \theta, m\right)$-quasisounivex at $y$, and for each $t \in \mathcal{L}, \phi_{t}$ is increasing and $\phi_{t}(0)=0$, where $\left\{\mathcal{L}_{1}, \mathcal{L}_{2}\right\}$ is a partition of $\mathcal{L}$;

(ii) for each $t \in(\mathcal{M} \backslash \mathcal{L})_{1}, \xi \rightarrow \Lambda_{t}(\xi, v, w)$ is strictly $\left(\mathcal{F}, \beta, \phi_{t}, \rho_{t}, \theta, m\right)$-pseudosounivex at $y$, for each $t \in(\mathcal{M} \backslash \mathcal{L})_{2}, \xi \rightarrow \Lambda_{t}(\xi, v, w)$ is 
$\left(\mathcal{F}, \beta, \phi_{t}, \rho_{t}, \theta, m\right)$-quasisounivex at $y$, and for each $t \in \mathcal{M} \backslash \mathcal{L}, \phi_{t} \quad$ is increasing and $\phi_{t}(0)=0$, where $\left\{(\mathcal{M} \backslash \mathcal{L})_{1},(\mathcal{M} \backslash \mathcal{L})_{2}\right\}$ is a partition of $\mathcal{M} \backslash \mathcal{L}$;

(iii) $\quad \sum_{t \in \mathcal{M}} \rho_{t}(x, y) \geq 0$ for all $x \in \mathbb{F}$;

(iv) $\quad \mathcal{L}_{1} \neq \emptyset,(\mathcal{M} \backslash \mathcal{L})_{1} \neq \emptyset$, or $\sum_{t \in \mathcal{M}} \rho_{t}(x, y)>0$.

Then, $\varphi(x) \geq \psi_{I I I}(\mathcal{S})$, where $\psi_{I I I}$ is the objective function of $(D I I I)$.

Proof (a): Suppose to the contrary that $\varphi(x)<\psi_{I I I}(\mathcal{S})$. This implies that

$D(y, u) f_{i}(x)-N(y, u) g_{i}(x)<0, ; i \in \underline{p}$.

Since $u \geq 0$ and $u \neq 0$, we see that for each $t \in \mathcal{L}$,

$\sum_{i \in I_{t}} u_{i}\left[f_{i}(x)-N(y, u) g_{i}(x)\right] \leq 0$.

Now, using this inequality, we see that

$$
\begin{aligned}
\Pi_{t}(x, y, u, v, w)= & \sum_{i \in I_{t}} u_{i}\left[D(y, u) f_{i}(x)-N(y, u) g_{i}(x)\right]+\sum_{j \in J_{t}} v_{j} G_{j}(x) \\
+ & \sum_{k \in K_{t}} w_{k} H_{k}(x) \leq \sum_{i \in I_{t}} u_{i}\left[D(y, u) f_{i}(x)-N(y, u) g_{i}(x)\right] \\
& (\text { by the primal feasibility of } x) \leq 0(\text { by }(4.6)) \\
\leq & \sum_{i \in I_{t}} u_{i}\left[D(y, u) f_{i}(y)-N(y, u) g_{i}(y)\right]+\sum_{j \in J_{t}} v_{j} G_{j}(y) \\
+ & \sum_{k \in K_{t}} w_{k} H_{k}(y)(\text { by }(4.3) \text { and the dual feasibility of } \mathcal{S}) \\
= & \Pi_{t}(y, y, u, v, w),
\end{aligned}
$$

and hence

$$
\phi_{t}\left(\Pi_{t}(x, y, u, v, w)-\Pi_{t}(y, y, u, v, w)\right) \leq 0,
$$

which in view of (i) implies that

$$
\begin{aligned}
& \mathcal{F}\left(x, y ; \beta(x, y)\left\{\sum_{i \in I_{t}} u_{i}\left[D(y, u) \nabla f_{i}(y)-N(y, u) \nabla g_{i}(y)\right]+\sum_{j \in J_{t}} v_{j} \nabla G_{j}(y)\right.\right. \\
& \left.\left.\quad+\sum_{k \in K_{t}} w_{k} \nabla H_{k}(y)\right\}\right)+\frac{1}{2}\left\langle z,\left\{\sum_{i \in I_{t}} u_{i}\left[D(y, u) \nabla^{2} f_{i}(y)-N(y, u) \nabla^{2} g_{i}(y)\right]\right.\right. \\
& \left.\left.\quad+\sum_{j \in J_{t}} v_{j} \nabla^{2} G_{j}(y)+\sum_{k \in K_{t}} w_{k} \nabla^{2} H_{k}(y)\right\} z\right\rangle<-\rho_{t}(x, y)\|\theta(x, y)\|^{m} .
\end{aligned}
$$

Summing over $t \in \mathcal{L}$ and using the sublinearity of $\mathcal{F}(x, y ; \cdot)$, we obtain

$$
\begin{aligned}
\mathcal{F} & \left(x, y ; \beta(x, y)\left\{\sum_{i=1}^{p} u_{i}\left[D(y, u) \nabla f_{i}(y)-N(y, u) \nabla g_{i}(y)\right]+\sum_{t \in \mathcal{L}}\left[\sum_{j \in J_{t}} v_{j} \nabla G_{j}(y)\right.\right.\right. \\
& \left.\left.\left.+\sum_{k \in K_{t}} w_{k} \nabla H_{k}(y)\right]\right\}\right)+\frac{1}{2}\left\langle z,\left\{\sum_{i=1}^{p} u_{i}\left[D(y, u) \nabla^{2} f_{i}(y)-N(y, u) \nabla^{2} g_{i}(y)\right]\right.\right. \\
& \left.\left.+\sum_{t \in \mathcal{L}}\left[\sum_{j \in J_{t}} v_{j} \nabla^{2} G_{j}(y)+\sum_{k \in K_{t}} w_{k} \nabla^{2} H_{k}(y)\right]\right\} z\right\rangle<-\sum_{t \in \mathcal{L}} \rho_{t}(x, y)\|\theta(x, y)\|^{m} .
\end{aligned}
$$

Proceeding as in the proof of Theorem 2.2, we get for each $t \in \mathcal{M} \backslash \mathcal{L}$,

$\Lambda_{t}(x, v, w) \leq \Lambda_{t}(y, v, w)$, and so

$\phi_{t}\left(\Lambda_{t}(x, v, w)-\Lambda_{t}(y, v, w)\right) \leq 0$,

which in view of (ii) implies that

$$
\begin{aligned}
& \mathcal{F}\left(x, y ; \beta(x, y)\left[\sum_{j \in J_{t}} v_{j} \nabla G_{j}(y)+\sum_{k \in K_{t}} w_{k} \nabla H_{k}(y)\right]\right)+\frac{1}{2}\left\langle z,\left[\sum_{j \in J_{t}} v_{j} \nabla^{2} G_{j}(y)\right.\right. \\
& \left.\left.\quad+\sum_{k \in K_{t}} w_{k} \nabla^{2} H_{k}(y)\right] z\right\rangle \leq-\rho_{t}(x, y)\|\theta(x, y)\|^{m}
\end{aligned}
$$

Summing over $t \in \mathcal{M} \backslash \mathcal{L}$ and using the sublinearity of $\mathcal{F}(x, y ; \cdot)$, we get

$$
\begin{aligned}
\mathcal{F}\left(x, y ; \beta(x, y) \sum_{t \in \mathcal{M} \backslash \mathcal{L}}\left[\sum_{j \in J_{t}} v_{j} \nabla G_{j}(y)+\sum_{k \in K_{t}} w_{k} \nabla H_{k}(y)\right]\right) \\
\quad+\frac{1}{2}\left\langle z, \sum_{t \in \mathcal{M} \backslash \mathcal{L}}\left[\sum_{j \in J_{t}} v_{j} \nabla^{2} G_{j}(y)+\sum_{k \in K_{t}} w_{k} \nabla^{2} H_{k}(y)\right] z\right\rangle \\
<-\sum_{t \in \mathcal{M} \backslash \mathcal{L}} \rho_{t}(x, y)\|\theta(x, y)\|^{m} .
\end{aligned}
$$

Now, combining (4.7) and (4.8) and using (iii), we obtain

$$
\begin{aligned}
\mathcal{F} & \left(x, y ; \beta(x, y)\left\{\sum_{i=1}^{p} u_{i}\left[D(y, u) \nabla f_{i}(y)-N(y, u) \nabla g_{i}(y)\right]+\sum_{j=1}^{q} v_{j} \nabla G_{j}(y)\right.\right. \\
& \left.\left.+\sum_{k=1}^{r} w_{k} \nabla H_{k}(y)\right\}\right)+\frac{1}{2}\left\langle z,\left\{\sum_{i=1}^{p} u_{i}\left[D(y, u) \nabla^{2} f_{i}(y)-N(y, u) \nabla^{2} g_{i}(y)\right]\right.\right. \\
& \left.\left.+\sum_{j=1}^{q} v_{j} \nabla^{2} G_{j}(y)+\sum_{k=1}^{r} w_{k} \nabla^{2} H_{k}(y)\right\} z\right\rangle \\
& <-\sum_{t \in \mathcal{M}} \rho_{t}(x, y)\|\theta(x, y)\|^{m} \leq 0 .
\end{aligned}
$$

Now, multiplying (4.1) by $\beta$, applying the sublinear function $\mathcal{F}(x, y ; \cdot)$ to both sides of the resulting equation, and then adding the equation to (4.2), we get

$$
\begin{aligned}
\mathcal{F} & \left(x, y ; \beta(x, y)\left\{\sum_{i=1}^{p} u_{i}\left[D(y, u) \nabla f_{i}(y)-N(y, u) \nabla g_{i}(y)\right]+\sum_{j=1}^{q} v_{j} \nabla G_{j}(y)\right.\right. \\
& \left.\left.+\sum_{k=1}^{r} w_{k} \nabla H_{k}(y)\right\}\right)+\frac{1}{2}\left\langle z,\left\{\sum_{i=1}^{p} u_{i}\left[D(y, u) \nabla^{2} f_{i}(y)-N(y, u) \nabla^{2} g_{i}(y)\right]\right.\right. \\
& \left.\left.+\sum_{j=1}^{q} v_{j} \nabla^{2} G_{j}(y)+\sum_{k=1}^{r} w_{k} \nabla^{2} H_{k}(y)\right\} z\right\rangle \geq 0,
\end{aligned}
$$

which contradicts (4.9). Therefore, we conclude that $\varphi(x) \geq \psi_{I I I}(\mathcal{S})$. (b) $-(\mathrm{g})$ : The proofs are similar to that of part (a).

Theorem 4.2 (Strong Duality) Let $x^{*}$ be a normal optimal solution of $(P)$ and assume that any one of the seven sets of conditions set forth in Theorem 4.1 is satisfied for all feasible solutions of (DIII). Then, for each $z^{*} \in C\left(x^{*}\right)$, there exist $u^{*}, v^{*}, w^{*}$, and $\lambda^{*}$, such that $\left(x^{*}, z^{*}, u^{*}, v^{*}\right)$ is an optimal solution of (DIII) and $\varphi\left(x^{*}\right)=\psi_{\text {III }}\left(x^{*}, z^{*}, u^{*}, v^{*}\right)$.

Proof The proof is similar to that of Theorem 2.2. 
The generalized duality models $(D I I I)$ and $(\tilde{D} I I I)$ subsume a great variety of special cases which can be identified explicitly by appropriate choices of the partitioning sets $\left\{I_{0}, I_{1}, \ldots, I_{\ell}\right\},\left\{J_{0}, J_{1}, \ldots, J_{M}\right\}$, and $\left\{K_{0}, K_{1}, \ldots, K_{M}\right\}$.

\section{Concluding remarks}

Remark 5.1 Using a direct nonparametric approach, in this paper, we have formulated six generalized secondorder parameter-free duality models for a discrete minmax fractional programming problem and established numerous duality results using a variety of generalized $(\mathcal{F}, \beta, \phi, \rho, \theta, m)$-sounivexity assumptions. Each one of the six duality models considered in this paper is, in fact, a family of dual problems whose members can easily be identified by appropriate choices of certain sets and functions. The generalized duality models and the related duality theorems collectively provide a vast number of new second-order dual problems and duality theorems for the principal minmax problem $(P)$ and its special cases designated as $(P 1)-(P 3)$ in Sect. 2. Furthermore, the style of presentation adopted in this paper as well as the main results derived here will prove useful in investigating other related classes of nonlinear programming problems and utilizing similar generalized convexity concepts. For example, employing similar techniques, one can investigate the second-order sufficient optimality and duality aspects of the following 'semiinfinite' minmax fractional programming problem:

$$
\text { Minimize } \max _{1 \leq i \leq p} \frac{f_{i}(x)}{g_{i}(x)}
$$

subject to

$$
\begin{aligned}
& G_{j}(x, t) \leq 0 \text { for all } \mathrm{t} \in T_{j}, j \in \underline{q} ; H_{k}(x, s)=0 \text { for } \\
& \text { all } \mathrm{s} \in S_{k}, k \in \underline{r} x \in X,
\end{aligned}
$$

where $X, f_{i}$, and $g_{i}, i \in p$, are as defined in the description of $(P)$, for each $j \in q$ and $\bar{k} \in \underline{r}, T_{j}$ and $S_{k}$ are compact subsets of complete metric spaces, for each $j \in \underline{q}, \xi \rightarrow G_{j}(\xi, t)$ is a real-valued function defined on $X$ for all $t \in T_{j}$, for each $k \in \underline{r}, \xi \rightarrow H_{k}(\xi, s)$ is a real-valued function defined on $X$ for all $s \in S_{k}$, for each $j \in \underline{q}$ and $k \in \underline{r}, t \rightarrow G_{j}(x, t)$ and $s \rightarrow H_{k}(x, s)$ are continuous real-valued functions defined, respectively, on $T_{j}$ and $S_{k}$ for all $x \in X$.

Remark 5.2 The generalized parametric duality model results, established in this paper applying generalized $(\mathcal{F}, \beta, \phi, \rho, \theta, m)$-sounivexity assumptions, can be generalized to the case of the generalized $\left(\mathcal{F}, \beta, \phi, h\left(x^{*}, z\right)\right.$, $\left.\kappa\left(x^{*}, z\right), \rho, \theta, m\right)$-sounivexity.
Definition 5.1 The function $f$ is said to be (strictly) $\left(\mathcal{F}, \beta, \phi, h\left(x^{*}, z\right), \kappa\left(x^{*}, z\right), \rho, \theta, m\right)$-sounivex at $x^{*}$ of higher order if there exist functions $\beta: X \times X \rightarrow \mathbb{R}_{+} \backslash\{0\} \equiv$ $(0, \infty), \phi: \mathbb{R} \rightarrow \mathbb{R}, \rho: X \times X \rightarrow \mathbb{R}, \theta: X \times X \rightarrow \mathbb{R}^{n}$, and a sublinear function $\mathcal{F}\left(x, x^{*} ; \cdot\right): \mathbb{R}^{n} \rightarrow \mathbb{R}$, such that for each $x \in X\left(x \neq x^{*}\right)$ and $z \in \mathbb{R}^{n}$,

$$
\begin{aligned}
\phi\left(f(x)-f\left(x^{*}\right)\right)(>) \geq & \mathcal{F}\left(x, x^{*} ; \beta\left(x, x^{*}\right)\left[\nabla_{z} \kappa\left(x^{*}, z\right)\right]\right) \\
& +\left\langle z, \nabla_{z} h\left(x^{*}, z\right)\right\rangle-h\left(x^{*}, z\right) \\
& +\rho\left(x, x^{*}\right)\left\|\theta\left(x, x^{*}\right)\right\|^{m},
\end{aligned}
$$

where $h, \kappa: \mathbb{R}^{n} \times \mathbb{R}^{n} \rightarrow \mathbb{R}^{n}$ are differentiable.

Acknowledgments The author is greatly indebted to the reviewer for all valuable comments leading to the revised version.

Open Access This article is distributed under the terms of the Creative Commons Attribution 4.0 International License (http://crea tivecommons.org/licenses/by/4.0/), which permits unrestricted use, distribution, and reproduction in any medium, provided you give appropriate credit to the original author(s) and the source, provide a link to the Creative Commons license, and indicate if changes were made.

\section{References}

1. Antczak, T.: Generalized fractional minimax programming with B-(p, r)-invexity. Comp. Math. Appl. 56, 1505-1525 (2008)

2. Craven, B.D., Mond, B.: On converse duality in nonlinear programming. Operat. Res. 19, 1075-1078 (1971)

3. Hanson, M.A.: Second order invexity and duality in mathematical programming. Opsearch 30, 313-320 (1993)

4. Mangasarian, O.L.: Second and higher-order duality in nonlinear programming. J. Math. Anal. Appl. 51, 607-620 (1975)

5. Mond, B.: Second order duality for nonlinear programs. Opsearch 11, 90-99 (1974)

6. Mond, B., Zhang, J.: Duality for multiobjective programming involving second-order V-invex functions. In: Glover, M., Jeyakumar, V. (eds.) Proceedings of the Optimization Miniconference II (B), pp. 89-100. University of New South Wales, Australia (1995)

7. Mond, B., Weir, T.: Generalized concavity and duality, Generalized in Optimization and Economics (S. Schaible and W. T. Ziemba, eds.), Academic Press, New York (1981) pp. 263-279

8. Verma, R.U.: Generalized B- $(b, \rho, \theta, \tilde{p}, \tilde{r})$-invexities and efficiency conditions for multiobjective fractional programming. Tbilisi Math. J. 8(2), 159-180 (2015)

9. Verma, R.U.: Generalized higher order $(\phi, \eta, \omega, \pi, \rho, \theta, \mathrm{m})$-invexities in parametric optimality conditions for discrete minmax fractional programming. J. Basic Appl. Sci. 12, 283-300 (2016)

10. Verma, R.U., Zalmai, G.J.: Second-order parametric optimality conditions in discrete minmax fractional programming. Commun. Appl. Nonlinear Anal. 23(3), 1-32 (2016)

11. Verma, R.U., Zalmai, G.J.: Generalized second-order parametric optimality conditions in discrete minmax fractional programming. Trans. Math. Program. Appl. 2(12), 1-20 (2014)

12. Verma, R.U., Zalmai, G.J.: Parameter-free duality in discrete minmax fractional programming based on second-order optimality conditions. Trans. Math. Program. Appl. 2(11), 1-37 (2014) 
13. Verma, R.U., Zalmai, G.J.: Generalized second-order parametric optimality conditions in semiinfinite discrete minmax fractional programming and second order $(F, \beta, \phi, \rho, \theta, \mathrm{m})$-univexity. Stat. Optim. Inform. Comp. 4, 15-29 (2016)

14. von Neumann, J.: A model of general economic equilibrium. Review Econ. Stud. 13, 1-9 (1945)

15. Wang, S.Y.: Second order necessary and sufficient conditions in multiobjective programming. Numer. Funct. Anal. Optim. 12, 237-252 (1991)

16. Werner, J.: Duality in generalized fractional programming. Intl. Ser. Numer. Anal. 84, 341-351 (1988)

17. Wolfe, P.: A duality theorem for nonlinear programming. Quart. Appl. Math. 19, 239-244 (1961)

18. Yang, X.: Generalized convex duality for multiobjective fractional programs. Opsearch 31, 155-163 (1994)

19. Yang, X.Q.: Second-order conditions in $C^{1,1}$ optimization with applications. Numer. Funct. Anal. Optim. 14, 621-632 (1993)

20. Yang, X.Q.: Second-order global optimality conditions of convex composite optimization. Math. Prog. 81, 327-347 (1998)
21. Yang, X.Q.: Second-order global optimality conditions for optimization problems. J. Global Optim. 30, 271-284 (2004)

22. Zalmai, G.J.: Hanson-Antczak type generalized $(\alpha, \beta, \gamma, \xi, \eta, \rho, \theta)$ $\mathrm{V}$-invex functions in semiinfinite multiobjective fractional programming Part II: First-order parametric duality models. Adv. Nonlinear Variat. Inequal. 16(2), 61-90 (2013)

23. Zalmai, G.J.: Hanson-Antczak type generalized $(\alpha, \beta, \gamma, \xi, \eta, \rho, \theta)$ $\mathrm{V}$-invex functions in semiinfinite multiobjective fractional programming Part III: Second-order parametric duality models. Adv. Nonlinear Variat. Inequal. 16(2), 91-126 (2013)

24. Zalmai, G.J.: Optimality conditions and duality models for a class of nonsmooth constrained fractional variational problems. Optimization 30, 15-51 (1994)

25. Zalmai, G.J.: Optimality principles and duality models for a class of continuous-time generalized fractional programming problems with operator constraints. J. Stat. Manag. Syst. 1, 61-100 (1998) 